\title{
Tarzan and chain: exploring the ICO jungle and evaluating design archetypes
}

\author{
Nina M. Bachmann ${ }^{1}$ (D) $\cdot$ Benedict Drasch ${ }^{2,3}$ (D) $\cdot$ Gilbert Fridgen $^{2,3,4}$ (D) Michael Miksch $^{1} \cdot$ Ferdinand Regner $^{1} \cdot$ \\ André Schweizer ${ }^{2,3}$. Nils Urbach ${ }^{2,3,5}$ (D)
}

Received: 16 July 2019 / Accepted: 1 February 2021 / Published online: 5 June 2021

(C) The Author(s) 2021

\begin{abstract}
The phenomenon of a blockchain use case called initial coin offering (ICO) is drawing increasing attention as a novel funding mechanism. ICO is a crowdfunding type that utilizes blockchain tokens to allow for truly peer-to-peer investments. Although more than $\$ 7 \mathrm{bn}$ has been raised globally via ICOs as at 2018, the concept and its implications are not yet entirely understood. The research lags behind in providing in-depth analyses of ICO designs and their long-term success. We address this research gap by developing an ICO taxonomy, applying a cluster analysis to identify prevailing ICO archetypes, and providing an outlook on the token value market performance for individual archetypes. We identify five ICO design archetypes and display their secondary market development from both a short-term and a long-term perspective. We contribute to an in-depth understanding of ICOs and their implications. Further, we offer practitioners tangible design and success indications for future ICOs.
\end{abstract}

Keywords Blockchain $\cdot$ ICO $\cdot$ Taxonomy $\cdot$ Archetypes $\cdot$ Success analysis

JEL classification $\mathrm{G} 15 \cdot \mathrm{G} 23 \cdot \mathrm{O} 33$

\section{Introduction}

Blockchain is a distributed ledger technology and enables decentralized and transactional data-sharing across a network

Responsible Editor: Roger Bons

During a large part of the research activities associated with this paper, Gilbert Fridgen was Professor at the University of Bayreuth and Deputy Director of both, the FIM Research Center and the Project Group Business and Information Systems Engineering of the Fraunhofer FIT.

Benedict Drasch

benedict.drasch@fim-rc.de; benedict.drasch@ fit.fraunhofer.de

Nina M. Bachmann

nina.bachmann@tum.de

Gilbert Fridgen

gilbert.fridgen@ fit.fraunhofer.de; gilbert.fridgen@ fim-rc.de;

gilbert.fridgen@uni.lu

Michael Miksch

michael.miksch@tum.de

Ferdinand Regner

ferdinand.regner@tum.de

André Schweizer

andre.schweizer@fit.fraunhofer.de; andre.schweizer@fim-rc.de of untrusted participants (Beck et al. 2016). The technology emerged with the development of Bitcoin in 2008 (Fanning and Centers 2016; Nakamoto 2008). Over the past few years, blockchain has evolved into a multipurpose technology that

Nils Urbach

nils.urbach@fit.fraunhofer.de; nils.urbach@ fim-rc.de; nils.urbach@fb3.fra-uas.de

1 FIM Research Center, University of Augsburg, Universitaetsstr. 12, 86159 Augsburg, Germany

2 Project Group Business \& Information Systems Engineering of Fraunhofer FIT, Wittelsbacherring 10, 95444 Bayreuth, Germany

3 FIM Research Center, University of Bayreuth, Wittelsbacherring 10, 95444 Bayreuth, Germany

4 SnT - Interdisciplinary Centre for Security, Reliability and Trust, University of Luxembourg, 29 Avenue John F. Kennedy, 1855 Luxembourg, Luxembourg

5 Frankfurt University of Applied Sciences, Nibelungenplatz 1, 60318 Frankfurt am Main, Germany 
has attracted interest of both practitioners and academics in a large number of use cases (Catalini and Gans 2018; Glaser 2017). Particularly, sales of blockchain-based digital tokens (initial coin offerings / ICOs) are attracting much attention - as a novel funding mechanism (Boreiko and Sahdev 2018; Chanson et al. 2018; Drasch et al. 2020; Schweizer et al. 2017). Despite regulatory uncertainty (Amsden and Schweizer 2018; Li and Mann 2018; Zetzsche et al. 2017), ICO fundraising has grown exponentially throughout 2016 (29 ICOs worth $\$ 90 \mathrm{~m}$ ), 2017 (875 ICOs worth $\$ 6227 \mathrm{~m}$ ), and 2018 (1253 ICOs worth $\$ 7812 \mathrm{~m}$ ) (ICODATA.IO 2019; Vigna et al. 2018). Although the value dropped significantly in 2019, the ICO phenomenon's novelty still raises a number of questions that remain open (Chanson et al. 2018).

In particular, a systematic understanding of what exactly constitutes an ICO is missing yet necessary to establish a shared knowledge base. Given that the inherent idea of ICOs is to provide open, global, and decentralized access to funding, regulation of ICOs presents a previously unknown challenge (Amsden and Schweizer 2018). Regulators and many governmental institutions have just begun to act in the mostly unregulated ICO market Bachmann et al. (2019). A major problem is that although there were first approaches of standardization, ICOs are still very heterogeneous (EFSA 2017). Further, market observations have shown that ICOs' likelihoods of long-term success (token market performance) differ significantly and may depend on ICO design parameters (Adhami et al. 2018; Amsden and Schweizer 2018; Boreiko and Sahdev 2018; Fisch 2019). In this study, we understand design parameters as the ICO issuer's choices when designing the ICO as a funding mechanism. These choices are comparable to the IPO issuers (i.e., share pricing mechanism, share allocation, date).

Similar to investments in cryptocurrencies (e.g., Bitcoin and Ethereum), it remains unclear how beneficial ICOs are in the short and long terms for both issuers and investors. Thus, an in-depth analysis of ICO design variations is necessary to better understand this phenomenon and to react appropriately from the economic, societal, and regulatory perspectives. Research needs to provide a systematic knowledge base (Beck et al. 2017), to identify relevant ICO design dimensions, to derive predominant archetypes, and to thoroughly analyze them. Yet, there have been very few scientific studies in the young research stream on ICOs. Boreiko and Sahdev (2018) provided an overview over the evolution of ICOs, and Chanson et al. (2018) compared ICOs to traditional crowdfunding mechanisms. Amsden and Schweizer (2018) as well as Fisch (2019) have begun to analyze potential factors that influence ICOs' likelihoods of success. Lee et al. (2018) used data during token sales and analyzed the information cascade within the investor crowd. However, although these studies represent first important steps, we lack a comprehensive and in-depth analysis of ICO archetypes and their likelihoods of success. To address this research gap, we define the following research questions:

1. What are the design parameters of ICOs as a novel funding approach?

2. Which ICO archetypes exist and what design parameters characterize them?

3. What performance characteristics differentiate the identified ICO archetypes?

We seek to answer these research questions in a multimethod approach, which contains three phases. We build on a manually compiled data sample of 131 ICOs collected from a wide array of sources, including in-depth information on ICO design parameter characteristics and publicly available crypto-market performance data. To answer research question 1 , we develop a taxonomy of empirically validated ICO design parameters. Taxonomies are well suited to structure the groundwork for emerging research fields and serve as the first step into systematization (Williams et al. 2008). We follow the established and well-recognized taxonomy development method proposed by Nickerson et al. (2013). To answer research question 2, we build on our taxonomy and perform a two-stage cluster analysis to inductively classify the 131 ICO cases (Aldenderfer and Blashfield 1984; Hair et al. 2013; Ketchen and Shook 1996), utilizing the taxonomy's 23 dimensions as clustering variables. As a result, we propose and evaluate five ICOs archetypes and their prevailing dimensions and characteristics. We answer research question 3 by analyzing the identified ICO archetypes' secondary market performance, following Smith + Crown's (2017) research approach. To provide an outlook on token market performance, we analyze, illustrate, and discuss the individuals' as well as the archetypes' average short-term and long-term developments.

Given that our results are based on a sample of 131 ICOs, we would like to highlight that our findings will need further research to evaluate their external validity. Research on the ICO market is a moving target, and further ICOs and new developments of included ICOs may reveal new information. However, on this limited basis, we are still confident that we can provide some interesting theoretical contributions and practical implications. We develop a taxonomy that contributes to the descriptive knowledge of the young research domain, laying the foundation for further research and higher theory in the area (Gregor 2006). Further, we propose empirically derived archetypes obtained from a sound clustering method. Owing to the given sample limitations, these archetypes can only provide a first understanding of the ICO phenomenon. However, we believe to propose potentially useful insights for individuals and economic or regulatory organizations. Also, the analysis of the ICO archetypes' long-term performance allows to understand some factors that may 
constitute a potentially successful ICO. We enable practitioners and researchers to get to a systematic understanding of this emerging phenomenon. Further, we allow practitioners to conclude on concrete design suggestions for potential future ICOs concerning existing archetypes.

\section{Foundations}

\section{Blockchain and smart contracts}

Blockchain is a computer protocol for decentralized and transactional data-sharing across a large network of untrusted participants (Xu et al. 2017). Public interest in the first generation of blockchain was sparked when its role as the basis for cryptocurrencies was discovered with the publication of the Bitcoin whitepaper by Satoshi Nakamoto in 2008 (Nakamoto 2008; Zohar 2015). A second generation of blockchains, such as Ethereum, came with a built-in Turing-complete programming language that also provided a general-purpose programmable infrastructure that enables the use of smart contracts (Buterin 2014). Smart contracts, a concept first introduced by Nick Szabo in 1994, refer to programs that are executed on a blockchain; these allow parties to securely transact with one another without trust, as the correct execution of these programs is enforced by a consensus protocol (Beck et al. 2016; Glaser 2017; Sillaber and Waltl 2017; Szabo 1997).

Another key characteristic is the creation and use of tokens (Buterin 2014). Tokens are defined as digital units of account that are transferable on the blockchain; they can serve several purposes, such as currency functions or grant access to a service (Glaser and Bezzenberger 2015; Schweizer et al. 2017). Using blockchain as a decentralized IT infrastructure, smart contracts to implement program logic, and tokens as transferable digital assets, a wide range of use cases have emerged (Conley 2017; Nærland et al. 2017), such as managing digital assets, implementing trust-free asset trade, issuing tokens and subcurrencies, and providing tokens in exchange for an investment (Buterin 2017; Nærland et al. 2017).

\section{Initial coin offering: combining blockchain and crowdfunding}

Blockchain combined with crowdfunding enables a new phenomenon: ICOs. The phenomenon was first called the Bitcoin model for crowdfunding in 2014 and described as a new business model for open-source software, where any participant in a blockchain protocol can participate anonymously in the funding, development, and revenue collection using tokens (Ravikant 2014). Recently, ICOs have become a popular alternative financing method for organizations (Arnold et al. 2019; Boreiko and Sahdev 2018; ICObench 2018; Li and Mann 2018; Schweizer et al. 2017). Instead of giving investors shares in a company, the general idea of is to distribute tokens as rewards for investments. Tokens' functionality depends on the implementation and differ between applications (e.g., granting access to a service or platform, voting rights on strategic decisions). As the distribution of tokens gives users partial ownership in a network and the possibility to trade the tokens on secondary markets, it incentivizes both joining the network early and benefiting from a potential appreciation of the token price (Ehrsam 2016). This new and completely decentralized approach relies solely on peer-topeer mechanisms and strongly contrasts to traditional crowdfunding, where the matchmaking process between campaign creators and potential investors is often established by crowdfunding platforms or banks serving as the intermediary (Danmayr 2014; Ehrsam 2016; Haas et al. 2015; Schweizer et al. 2017). Currently, ICOs are used to fund the development of blockchain-related projects, such as new protocols or apps. Smart contracts enable the funding in advance, even before the de facto start of a project (Ehrsam 2016; Kuo Chuen 2017). According to the venture capitalist Ehrsam, the ICO model to fund projects in advance can also help to overcome the classic chicken and egg problem for networks (Ehrsam 2016).

\section{The status quo of initial coin offering success analysis}

Although ICOs are a very recent phenomenon and are associated with high uncertainty concerning their development, market acceptance, and validity, various organizations (particularly startups) prefer ICOs over traditional funding mechanisms (Adhami et al. 2018). Research has only begun to address this uncertainty by analyzing and evaluating ICOs' success. Owing to the various ICO design options, it is hard to define ICO success and associated measures, which differ significantly between approaches.

Adhami et al. (2018) analyzed the determinants of a successful ICO campaign and found that success is more likely if the source code is available and when a token pre-sale is organized. Further, the authors argue that ICOs 'fail' for various reasons, such as failing the minimum funding goal, failing owing to a security flaw, low funding that results from a de facto or a perceived scam, or the project promoters halting the crowdsale (Adhami et al. 2018). Amsden and Schweizer (2018) argued that the strongest measure of ICO success is whether the token is subsequently listed and traded on an exchange platform. Their approach builds on the assumption that exchange platforms have sufficient mechanisms (e.g., due diligences) in place, and only list legit and promising tokens in order to maintain their own reputation. Boreiko and Sahdev (2018) proposed a diverging understanding of and approach to measuring ICO success. The authors follow a two-step approach: First, they exclude fraudulent, postponed, dubious, or cancelled ICO campaigns. Second, they classify ICOs into 
top, failed, or average ICOs. In their approach, top ICOs reach their funding limit before the end of the campaign (hard caps), or raise more than the third quartile of all capped ICOs (no hard cap), while failed ICOs are those that raise less than the self-imposed minimum, and average ICOs summarize all ICOs that don't fit in the first or the second cluster.

Also, empirical research into ICOs is growing: Howell et al. (2018) documented different yet key features of the ICO structure in practice. Benedetti and Kostovetsky (2018) reported evidence of significant ICO underpricing, and resulting high returns, which are also consistent with high compensation for high investment risk (Benedetti and Kostovetsky 2018; Momtaz 2018). Further, Momtaz (2018) found that the management quality and the ICO profile are positively correlated with the funding amount and returns, whereas highly visionary projects had a negative effect. Lee et al. (2018) used novel data during token sales and found that the wisdom of the crowd overcomes the information asymmetry associated with an ICO. This proceeds via a number of informed investors who verify the quality of the underlying startup, and the crowd who then harnesses the wisdom during the fundraising period. Hu et al. (2018) analyzed secondary market returns and correlations, providing investment characteristics of 64 ICOs. Fisch (2019) drew on signaling theory, applying a frequently used approach to indicate investment success in traditional entrepreneurial finance (Ahlers et al. 2015; Mollick 2014) and business angel investments (Clercq and Dimov 2008; Cumming et al. 2005). According to Fisch's (2019) results, signals such as technical whitepapers and high-quality source codes increase both the amount raised and ICOs' likelihood of success.

These approaches are first valid steps toward a better understanding of evaluations of ICO success. However, while previous studies such as Fisch (2019; 423 ICOs from 2016 to 2018) and Howell et al. (2018; 1520 ICOs from 2017 to 2019) relied on larger data samples, the observed ICO characteristics were limited in their number and their coverage of ICO design parameters. Further, previous studies considered the crypto market as a whole, and none of the studies analyzed ICOs characteristics as well as the performance of ICOs based on an extensive differentiation of their design parameters. By building our performance analysis on an empirically obtained taxonomy with 66 characteristics, we aim to enrich existing literature by exploring determinants of ICOs which have not yet been included in previous studies. Simultaneously, by grouping the ICOs into archetypes, we aim to provide a holistic view on ICOs in order to complement previous perspectives which studied the impact of single variables on ICO performance. In summary, we seek to complement existing knowledge with our study, relying on a multitude of indepth ICO design characteristics and a simultaneous consideration of the crypto-market's development. The blockchain research organization Smith + Crown proposes a first procedure that included this perspective via comparing the performance of a specific token to Bitcoin and Ethereum (Smith + Crown 2017). Comparing the token to the two biggest cryptocurrencies seems to be a valid approach, since Bitcoin and Ethereum have proven to be indicators of the overall market performance.

\section{Research approach: three phases toward an understanding of ICOs}

We will now provide an overview over our multi-method research approach which consists of three phases to reflect our three research questions. In Table 1, we outline the three research phases. We conduct these iteratively and in a partly overlapping way to cater for this research field's dynamics. Particularly, whenever we identified novel developments or emerging ICO design parameters, we updated our previously developed research artifacts. This approach is in line with recent work by various researchers in the IS domain (Beck et al. 2016; Nickerson et al. 2013; Schweizer et al. 2017).

In phase 1, we developed a taxonomy as a first step toward structuring the emerging research domain of ICOs and established a foundation for the subsequent research activities. In phase 2, we performed a two-stage cluster analysis in line with the IS literature and the exploratory research setting to identify meaningful ICO archetypes (Haas et al. 2014; Malhotra et al. 2005; Püschel et al. 2016). In phase 3, we utilized the findings from phases 1 and 2 and applied Smith + Crown's (2017) market performance analysis to visualize the ICO archetypes' market development. In the next section, we will provide details on the data collection process, and then go on by describing each phase's methods and results in more detail.

\section{Data}

Our 3-phase research approach requires extensive information for each ICO. However, a reliable, objective, and universal database does not yet exist (Fisch 2018). Thus, we first extracted a list of ICOs from Coindesk's ICO Tracker (Coindesk.com 2018) and retrieved 815 ICOs from various industries and geographical regions between January 2013 and December 2018. Coindesk is a comparatively reputable and comprehensive data source (Adhami et al. 2018; Chanson et al. 2018) for ICOs. We conducted an iterative procedure to compile our own database by gradually including ICO cases. For each of the iterations, we randomly assembled ICOs from the list of 815 , and gathered information on the ICOs' design parameters. Given the exploratory and iterative nature of the taxonomy development, we kept only cases with exhaustive information available. This means, we iteratively had to drop a case as soon as we could not find one information that we had 
Table 1 Three-phase research approach

\begin{tabular}{|c|c|c|c|}
\hline & Research phase 1 & Research phase 2 & Research phase 3 \\
\hline $\begin{array}{l}\text { Research } \\
\text { question }\end{array}$ & - What are the design parameters of ICOs? & $\begin{array}{l}\text { - Which ICO archetypes exist and what design } \\
\text { parameters characterize them? }\end{array}$ & $\begin{array}{l}\text { - What performance characteristics } \\
\text { differentiate the identified ICO archetypes? }\end{array}$ \\
\hline Method & $\begin{array}{l}\text { - Taxonomy development following } \\
\text { Nickerson et al. (2013): Iterative taxon- } \\
\text { omy development with both } \\
\text { empirical-to-conceptual and } \\
\text { conceptual-to-empirical iterations }\end{array}$ & $\begin{array}{l}\text { - Two-stage cluster analysis based on } \\
\text { Balijepally et al. (2011): Hierarchical clus- } \\
\text { tering using Ward's methods and nonhier- } \\
\text { archical clustering using the k-modes al- } \\
\text { gorithm } \\
\text { - Validation: Pearson's } \chi 2 \text { tests and Cramer's } \\
\text { V index, pairwise post hoc tests }\end{array}$ & $\begin{array}{l}\text { - Success indication analysis based on Smith + } \\
\text { Crown (2017): Analysis of the secondary } \\
\text { market performance of individual ICOs and } \\
\text { of the archetypes for the 1-month, 6-month, } \\
\text { and 12-month intervals }\end{array}$ \\
\hline Data & $\begin{array}{l}\text { - Qualitative data on } 131 \text { ICOs gathered } \\
\text { from whitepapers and additional } \\
\text { sources, including legal term sheets, } \\
\text { media releases, and websites } \\
\text { - Interviews with six experts }\end{array}$ & $\begin{array}{l}\text { Building on the data from phase 1: } \\
\text { - Clustering objects: } 131 \text { ICOs } \\
\text { - Clustering variables: the taxonomy's } \\
\text { dimensions ( } n=23 \text { ) with the respective } \\
\text { characteristics as values }\end{array}$ & $\begin{array}{l}\text { Building on the data from phases } 1 \text { and } 2 \text { : } \\
\text { - Token sale market performance data for } 112 \\
\text { real-world ICOs } \\
\text { - Market performance data for Bitcoin and } \\
\text { Ethereum }\end{array}$ \\
\hline Result & $\begin{array}{l}\text { - Literature-based, empirically shaped, } \\
\text { and evaluated taxonomy for ICO design } \\
\text { parameters with } 23 \text { dimensions and } 66 \\
\text { characteristics }\end{array}$ & $\begin{array}{l}\text { - Five statistically verified ICO archetypes } \\
\text { based on their design parameters }\end{array}$ & $\begin{array}{l}\text { - Visualization and discussion of the } \\
\text { archetype's performance }\end{array}$ \\
\hline
\end{tabular}

considered relevant in previous cases. In iteration 1, we went through 315 randomly selected cases. After the first iteration, our database contained 84 ICOs for which sufficiently exhaustive information was available. In the second iteration, we randomly selected 200 ICOs from the remaining 500 cases. In this iteration, we added another 47 complete cases. In sum, we analyzed 515 cases to manually gather our dataset of 131 ICOs. During the two iterations, two researchers collected and classified each ICO case independently, using insights from whitepapers, other documents (e.g., legal term sheets, media releases, and specific websites to inform about an ICO), and smart contract code where available. In case of disagreements between the two researchers, they discussed the issue until they achieved convergence. We acknowledge that this sample presents a limitation in this study: the sample shapes our taxonomy (first research phase) and thereby impacts subsequent research phases. However, owing to the high cost of collecting the information, we stopped our data collection after these 131 complete cases because, at the end of the sampling, no new information was obtained from adding ICOs. This concept is described by Morse (1995) as saturation, or data adequacy. Thus, in our view, the sample serves as a sound foundation for our purpose. Further, for data triangulation, and since ICOs are a wholly novel phenomenon, we collected additional primary data in the form of interviews with experts (cf. Table 2) (Perks et al. 2005). We chose a semi-structured interview approach to assure comparability and to preserve explorativeness (Yin 2017). The interviews lasted between 30 and $45 \mathrm{~min}$. We recorded and then analyzed them according to scientific standards (Dexter 2006).

Finally, to be able to answer our third research question, we additionally collected publicly available performance data for our data sample of 131 ICOs. Of the original 131 ICOs, 19 were never publicly listed, resulting in 112 remaining cases for the subsequent analysis in our study. To account for differing patterns over time (i.e., short-term rallies and long-term development), we collected data on the short-term (1-month), medium-term (6-month), and long-term (12-month) intervals wherever available.

\section{Methods and results}

\section{Research phase 1: ICO taxonomy development}

A taxonomy is the result of a design science research approach and can therefore be viewed as an artifact that consists of dimensions that contain characteristics that are mutually exclusive and collectively exhaustive (Nickerson et al. 2010). A taxonomy seeks to lay the foundation for further research by systematically classifying characteristics of objects of interest, fostering understanding of a phenomenon (Glass and Vessey 1995). The classification process' focus allows for a systematic examination of the general principles and issues that underlie a classification scheme. Equally important, taxonomies can help to predict future development areas, similar to the periodic table, which predicted the existence of elements decades before they could be isolated (Glass and Vessey 1995). Multitudes of scientific studies have successfully relied on the creation or use of taxonomies to lay the groundwork for emerging research fields. Recent examples include explorative studies on cooperation between banks and FinTechs (Drasch et al. 2018), cloud networks (Keller and König 2014), decentralized consensus systems (Glaser and Bezzenberger 2015), smart things (Püschel et al. 2016), agile IT setups (Jöhnk et al. 2017), and blockchain-based systems 
(Xu et al. 2017). In line with these role models, we follow the iterative design-oriented taxonomy development method proposed by Nickerson et al. (2013), which goes beyond the traditional approach proposed by Bailey (1984). It integrates conceptual and empirical perspectives into one comprehensive method, fostering the iterative use of both paradigms (Nickerson et al. 2013). The taxonomy development method has seven steps: (1) determination of a meta-characteristic, (2) determination of ending conditions, (3) choice of approach (i.e., empirical-to-conceptual or conceptual-to-empirical), (4) conceptualization of characteristics and dimensions, (5) examination of objects, (6) design (i.e., creation or revision of the taxonomy), and (7) testing of the ending conditions. While the researcher chooses the meta-characteristic and the ending conditions at the start of the development process, several iterations of taxonomy design and improvement follow (steps 3 to $6)$.

We defined our meta-characteristic as follows (step 1): Design parameters and characteristics of ICOs as a novel crowdfunding type. Further (step 2), as our ending conditions, we define the eight objective and five subjective ending conditions $^{1}$ as proposed by Nickerson et al. (2013). We then carried out reciprocal empirical-to-conceptual and conceptual-toempirical iterations to develop the taxonomy (steps 3 to 7). In empirical-to-conceptual iterations, we used a subset of our ICO cases, examined them in detail to identify characteristics, and, subsequently, grouped the characteristics into distinct dimensions. The grouping step involves the creation of labels for sets of related characteristics (Bailey 1994). In conceptualto-empirical iterations, we deducted characteristics and dimensions based on literature, for example on auction theory, IPO processes, and crowdfunding, since this literature promises the identification of dimensions and characteristics relevant to our meta-characteristic. We then examined our ICO cases to verify these characteristics and dimensions' applicability. Non-appropriate dimensions are eliminated. After each iteration, we evaluated whether the current state of the taxonomy meets the ending conditions. During later iterations, we conduct this evaluation with the help of expert interviews (see Data section, Table 2), discussing the current state of the taxonomy. During the interviews, we thoroughly went through each dimension of the current taxonomy and discussed the integrity concerning both objective and subjective ending conditions (Nickerson et al. 2013). This allowed us to evaluate the proposed taxonomy based on real-world experience (empirical-to-conceptual) (Schultze and Avital 2011). After 14 iterations, our taxonomy met the determined ending conditions.

\footnotetext{
${ }^{1}$ Among others, the fundamental objective ending conditions include that all dimensions are mutually exclusive and collectively exhaustive, and that all objects in the sample have been examined (Nickerson et al. 2013). Subjective ending conditions are conciseness, robustness, comprehensiveness, extendibility and explanatority. For reasons of brevity, we refer for further details to Nickerson et al. (2013) and Fridgen et al (2018).
}

Our research artifact is shown in Table 3. Overall, our taxonomy consists of 23 relevant dimensions, encompassing 66 characteristics that we defined according to the specified meta-characteristic. A detailed definition of the dimensions and characteristics can be found in Fridgen et al. (2018) and Bachmann et al. (2019).

\section{Research phase 2: identifying ICO archetypes}

To identify prevailing ICO archetypes, we performed a cluster analysis, in line with the IS literature and the exploratory research setting (Haas et al. 2014; Malhotra et al. 2005; Püschel et al. 2016). A cluster analysis is a statistical technique that seeks to group similar entities into various clusters. It minimizes the within-group variance while maximizing the between-group variance (Aldenderfer and Blashfield 1984; Hair et al. 2013). Generally, cluster analysis is applicable to describe generic archetypes of entities (Everitt et al. 2011; Hair et al. 2013). An analysis of 55 IS articles indicated that this method is often chosen to classify observations of specific objects of interest (Balijepally et al. 2011). Our cluster analysis consists of three steps: (i) Selection of the clustering variables; (ii) determination of an appropriate cluster algorithm; and (iii) confirmation of the results' reliability and validity through the application of statistical methods.

The selection of clustering variables is a fundamental step in cluster analysis, because it strongly affects the outcome (Punj and Stewart 1983). Following a deductive approach (Ketchen et al. 1993), the chosen variables must be closely linked to extant theory (Ketchen and Shook 1996), which is why selected our taxonomy's both empirically and conceptually developed dimensions as clustering variables. Note that this choice may potentially lead to an overweighting of underlying constructs among the dimensions if clustering variables are correlated (which we expect when searching for archetypes) (Ketchen and Shook 1996). We therefore conducted a multiple correspondence analysis (MCA). We obtained low eigenvalues for the resulting factors, which is why we kept all 23 taxonomy dimensions as clustering variables.

After the selection of the cluster variables, we selected an appropriate clustering algorithm. We applied a two-stage clustering algorithm, combining advantages of both

Table 2 Expert interviews

Current position and experience

1 Board member of cryptocurrency community - ICO investor

2 Academic researcher - ICO advisor, ICO researcher

3 Consultant - ICO advisor

4 Attorney - ICO advisor

5 Academic researcher - ICO researcher

6 Academic researcher - ICO researcher 
Table 3 Taxonomy of ICOs

\begin{tabular}{|c|c|c|c|c|c|c|}
\hline Dimension & Characteristics & & & & & \\
\hline Token implementation level & on-chain & & native & & sidechain & \\
\hline Token purpose/type & usage & work & funding & staking & equity security & non-equity security \\
\hline Token supply growth & fixed & & adaptive inflati & & fixed inflation & \\
\hline Token supply cap & capped & & & uncapped & & \\
\hline Token burning & yes & & & no & & \\
\hline Token distribution deferral & yes & & & no & & \\
\hline Token holder voting rights & yes & & & no & & \\
\hline Issuing legal structure & foundation & & & limited & & \\
\hline Team company token share & minority & & majority & & half & \\
\hline Team lockup period & no & & single period & & multiple periods & \\
\hline Pre-sale before ICO & no & private & & public & both & \\
\hline Pre-sale discount & yes & & & no & & \\
\hline Planned occurrence & multiple rounds & & single round & & unspecified & \\
\hline Registration needed & yes & & & no & & \\
\hline Eligibility restrictions & none & geographic & & accreditation & multiple & \\
\hline Purchase amount limit & none & minimum & & maximum & both & \\
\hline Auction mechanism & yes & & & no & & \\
\hline Sales price & fixed & & & floating & & \\
\hline Price fixing currency & crypto & & & fiat & & \\
\hline Funding currency & crypto & & both & & none & \\
\hline Funding cap & none & hard cap & & soft cap & multiple & \\
\hline Time horizon & block time & & fixed date & & open end & \\
\hline Time-based discount & none & & single rate & & multiple rates & \\
\hline
\end{tabular}

nonhierarchical and hierarchical procedures, to improve the clustering performance and to get more accurate and reproduceable results (Aldenderfer and Blashfield 1984; Ketchen and Shook 1996; Milligan and Sokol 1980; Punj and Stewart 1983). This approach is also supported by various IS research experts (Balijepally et al. 2011). In this the twostage clustering process, the clustering algorithm starts with a hierarchical clustering. We applied Ward's method, which is the most commonly applied algorithm among the hierarchical methods (Balijepally et al. 2011) owing to the production of reliable cluster results (Haas et al. 2014; Malhotra et al. 2005; van de Vrande et al. 2009). For the distance measure between categorical data points, the literature recommends using the Jaccard, the simple matching, and the Dice distance measures (Berkhin 2006; Finch 2005). We tested different measures and found that they all produce very similar results (Haas et al. 2014). We then inspected the dendrograms and the scree-plots that result from the hierarchical clustering with the Jaccard, the simple matching, and the Dice distance measures, to determine the appropriate number of clusters (Aldenderfer and Blashfield 1984). This step revealed that five clusters represent the favorable number of clusters, since any additional cluster would not significantly lower the total within-cluster sum of squares. The clustering dendrogram with the Jaccard
Distance Measure is presented in Fig. 1. Further, we computed the average silhouette width and the gap statistic (Tibshirani et al. 2001) which both confirmed the five-cluster solution.

As a second stage in the two-stage clustering algorithm, we then conducted nonhierarchical clustering. IS researchers widely use the k-means approach with Euclidean distance measure (Balijepally et al. 2011). However, research indicates that $\mathrm{k}$-means is not the optimal approach to process categorical data, since Euclidean distances are not meaningful on a discrete sample space (Chaturvedi et al. 2001). Thus, Huang (1998) proposed a nonhierarchical clustering algorithm called k-modes, using a simple dissimilarity measure and substituting the means of the clusters with modes (Chaturvedi et al. 2001; Xu et al. 2017). K-modes, similar to k-means, requires the pre-specification of the number of clusters, Herein, we use the results from the first stage as input, and therefore set the number of clusters to five. The application of the k-modes algorithm to the dataset resulted in our five final clusters which we then define as our five archetypes.

Finally, for validation purposes, Hair et al. (2013) suggested finding significant differences between the clusters among their respective characteristics of the clustering variables (i.e., dimensions). We therefore conducted a crosstabulation analysis to identify which variables significantly 
contribute to the differentiation of clusters (i.e., archetypes) (Everitt et al. 2011). We applied Pearson's $\chi^{2}$ and Cramer's $\mathrm{V}$, which measure for a relationship's strength, to analyze global differences across all clusters in the categorical data points (Haas et al. 2014; Malhotra et al. 2005). We then ran post hoc tests for a pairwise comparison of single clusters to one another, using Pearson's $\chi^{2}$ with correction for alpha inflation (Bonferroni style).

The main results of the cluster analysis are summarized and displayed in Table 4. First, based on the cluster analysis, we propose five prevailing ICO archetypes. Second, the results also indicate the validity of the taxonomy as the basis of our analysis, since the values indicate significant contributions of the characteristics. The $\chi^{2}$ reported significant values for most cluster variables, and the Cramer's V reported medium to strong association. The exceptions reflected some sales terms variables, i.e., the funding currency and the fixing of the price, closely related to the auction mechanism, as well as two timerelated sales terms. Little information was gained from these variables, and there was low variation among clusters. We also conducted the clustering without these variables and received nearly identical results. Thus, we kept the variables in the taxonomy so as to avoid information loss (Soh and Markus 2002), since we perceive them as important dimensions in the characterization.

\section{Research phase 3: the ICO archetype performance analysis}

Research phase 3 investigated the development of the archetypes to provide an outlook on both single cases and on the overall archetype development. In line with existing studies, this research phase drew on the token value performance in the secondary market (Amsden and Schweizer 2018; Smith + Crown 2017). Smith + Crown's (2017) approach considers

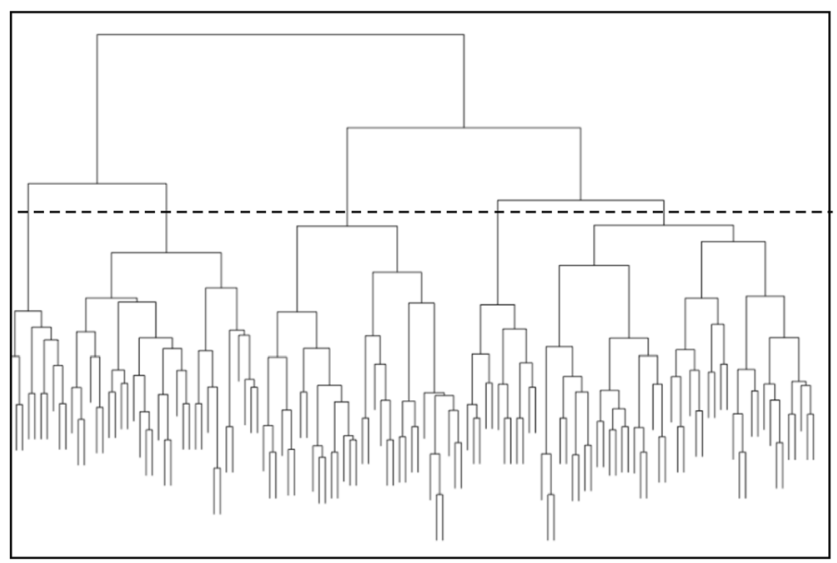

Fig. 1 Dendrogram after hierarchical clustering with the Jaccard distance measure the volatility and the idiosyncrasies of the crypto market and combines and extends previous research attempts. It extends Amsden and Schweizer's (2018) approach, since it not only evaluates if a token is listed on an exchange platform, but also analyzes the performance over time. Further, it integrates Boreiko and Sahdev's (2018) idea by excluding dubious ICOs prior to the analysis.

Since the cryptocurrency 'gold rush' heavily influenced the entire token market development between 2016 and 2018 (Amsden and Schweizer 2018), there is also the necessity to account for these market specificities. Thus, we compared the ICOs' market performances to those of cryptocurrency with identical time intervals. Bitcoin and Ethereum served as the main representatives. Combining these perspectives and following our research objectives, the following analysis consists of four steps and is structured as follows. In step 1, as a basis for the subsequent steps, we visualize the development of single ICOs with regard to their archetype. In step 2 , we aggregate the return rate for all ICOs within one archetype to compute the mean return rate for the overall archetype. This allows for the indicative analysis of the short-term, medium-term, and long-term development of each archetype. In step 3, we compare the mean return rate for each archetype to the Bitcoin and Ethereum return rate during the same period. Thus, the development is more realistic and accounts for market specificities. Finally, in step 4, we calculate the relative and absolute number of ICO cases in each archetype that performed better/worse than the mean of our entire sample, and that performed better/worse than Bitcoin and Ethereum in the identical period. As a first step, an outlook on the development of single ICOs with regard to their archetype is provided. Figures 2, 3, and 4 illustrate the 1month, 6-month, and 12-month development of all 112 ICOs. The archetype assignment is visualized by color and the ICOs are chronically plotted according to their issuing date.

In step 2, we calculated the mean return rate for each archetype (Table 5). We aggregated these three return rates for each archetype and each interval as follows. We calculated each ICO's token value development for the aforementioned periods. We then aggregated the 1-month, 6-month, and 12month intervals and calculated a mean return rate for the archetype per interval. Figure 5 provides an exemplary calculation, where we aggregated an exemplary archetype which consists of three ICOs.

The results indicated a positive and increasing secondary market performance in the 1-month, 6-month, and 12-month intervals for Archetypes 1 and 2. Archetype 4 indicated an increasingly negative development throughout all three intervals. Overall, the sample's return rate increased from the 1month to the 6-month to the 12-month interval. However (see Table 5), since the subsample sizes for Archetypes 3 and 5 were very small $(<20)$, we focused on Archetypes 1, 2, and 4 


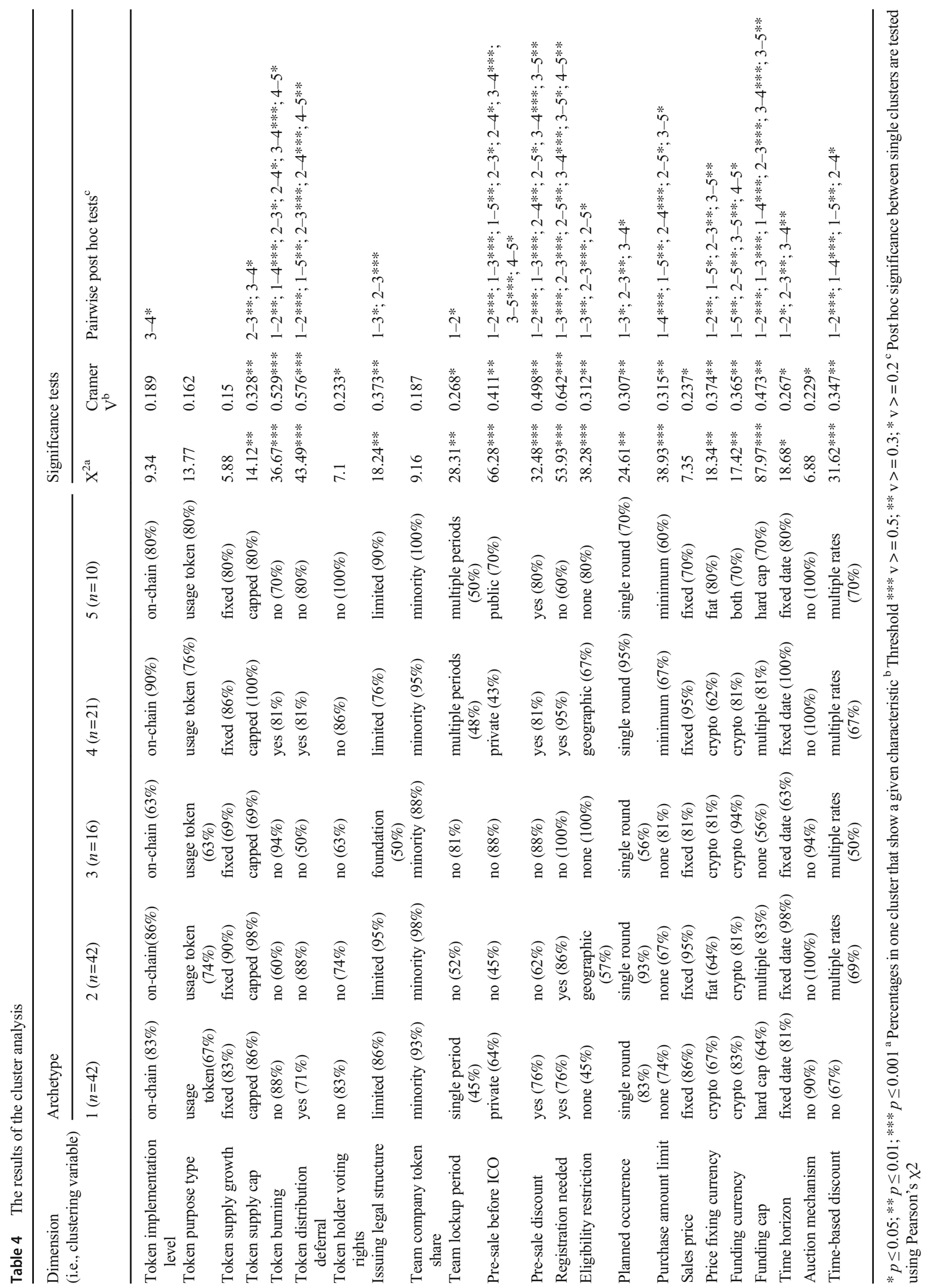


Fig. 2 Visualization of the singular ICO short-term absolute performance (1 month; no aggregation, ICOs assigned to their initial issuing date)

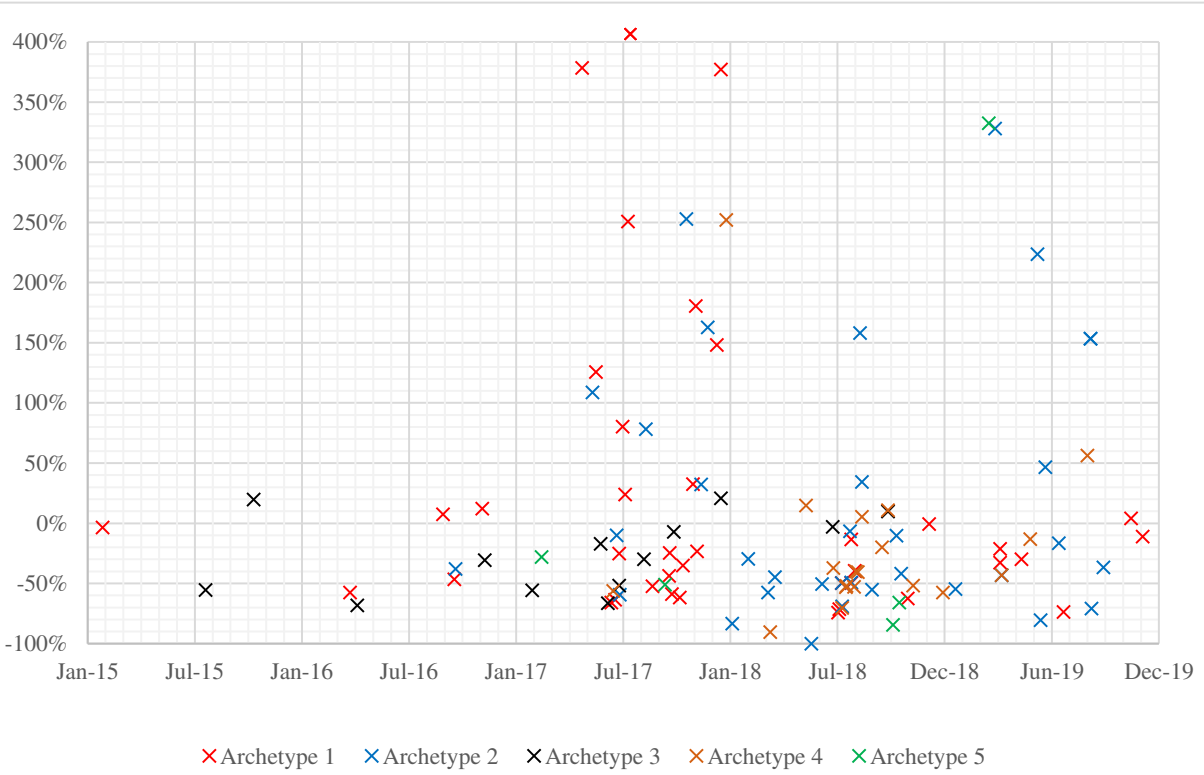

for the further analysis and do not report any quantitative analyses for Archetypes 3 and 5 (Tables 6 and 7).

Step 3: To account for the market volatility in the cryptocurrency market during the past months, we analyzed the mean performance of the ICO archetype along with the two most important cryptocurrency values in the same period (Table 6).

The results revealed that, in the short term (1-month return rate), Archetype 1 and 2 ICOs had better average returns than Bitcoin and Ethereum in the same periods. Archetype 4 ICOs revealed negative short-term average return rates, while both Bitcoin and Ethereum had slightly better return rates. In the 6month return rate comparison, Archetype 1 and 2 ICOs had more positive average return rates than the market representatives. The return rates for Archetype 4 and Ethereum remained negative, whereas Bitcoin turned from almost negative (1-month interval) to positive (6-month interval) in the same period. In the long term (12-month interval), the return rates for Archetype 1 and 2 ICOs continued to increase and outperformed Bitcoin and Ethereum. The average return rate for Archetype 4 ICOs remained negative and decreased even further. In the same period, Bitcoin increased its return, while Ethereum remained at a similar negative level. A key factor to consider is the all-time high of Ethereum in January 2018 (January 13, 2018: \$1385.02) compared to one year before (January 13, 2017: \$9.78).

Step 4: Building on the results from step 3, we compared the number of ICOs for every archetype. Thus, we counted the
Fig. 3 Visualization of the singular ICO medium-term absolute performance (6 months; no aggregation, ICOs assigned to their initial issuing date)

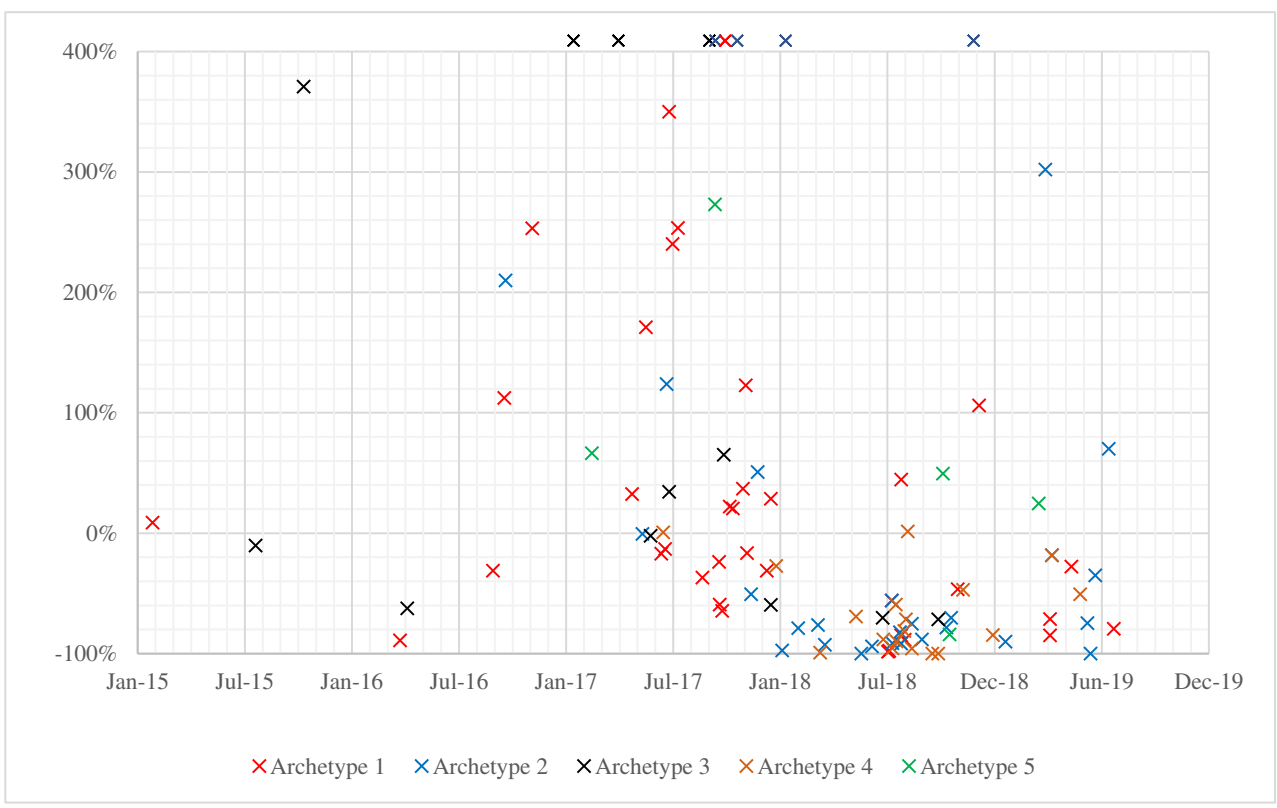


Fig. 4 Visualization of the singular ICO long-term absolute performance (12 months; no aggregation, ICOs assigned to their initial issuing date)

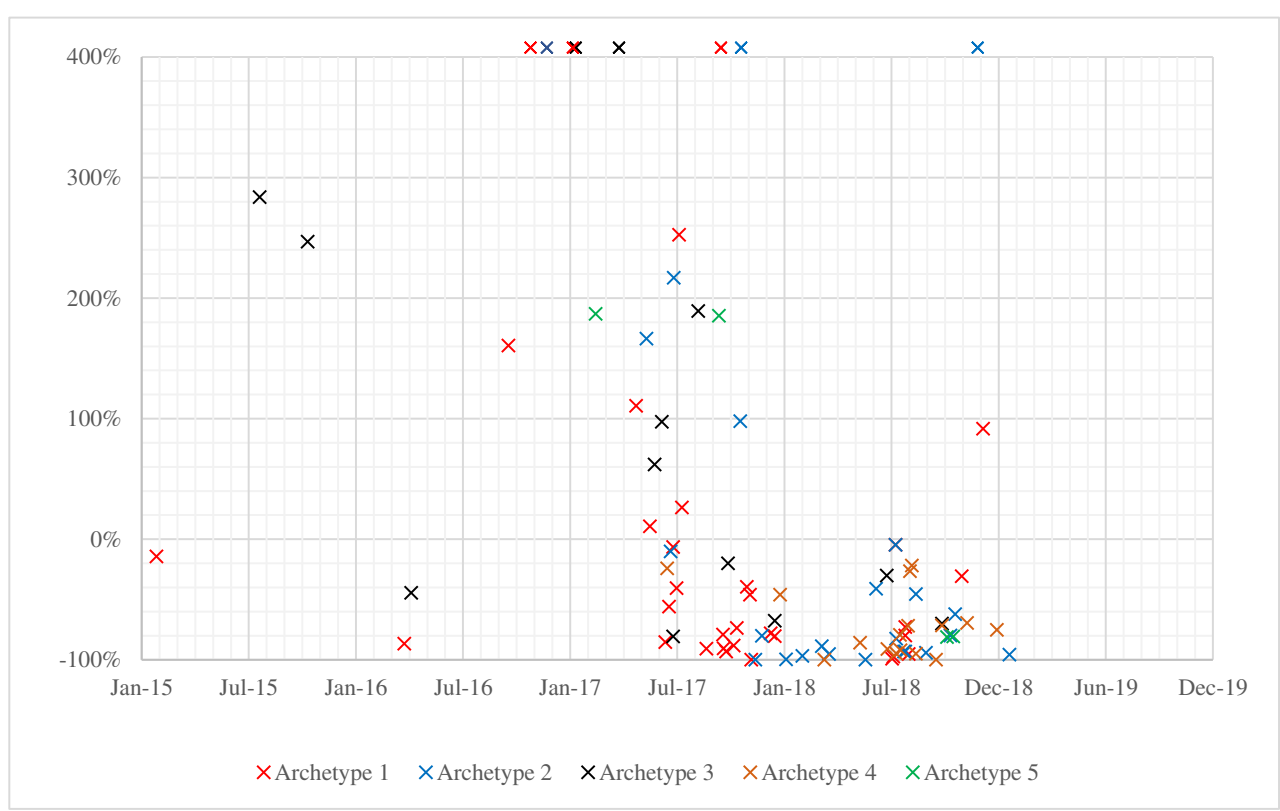

Table 5 The archetype token value return rates and the number of cases (1, 6, and 12 months after the ICO)

\begin{tabular}{|c|c|c|c|c|c|c|c|}
\hline \multirow[b]{2}{*}{ Archetype 1} & \multirow{2}{*}{$\begin{array}{l}\begin{array}{l}\text { Total cases } \\
\text { (excluded) }\end{array} \\
42(3)\end{array}$} & \multicolumn{2}{|c|}{$\begin{array}{l}\text { 1-month return rate in } \% \\
\text { (number of cases) }\end{array}$} & \multicolumn{2}{|c|}{$\begin{array}{l}\text { 6-month return rate in \% } \\
\text { (number of cases) }\end{array}$} & \multicolumn{2}{|c|}{$\begin{array}{l}\text { 12-month return rate in } \% \\
\text { (number of cases) }\end{array}$} \\
\hline & & $43.8 \%$ & (39) & $204.2 \%$ & (37) & $417.6 \%$ & (33) \\
\hline Archetype 2 & $42(8)$ & $19.8 \%$ & (34) & $102.5 \%$ & (30) & $135.4 \%$ & (24) \\
\hline Archetype 3 & $16(1)$ & $-22.5 \%$ & (15) & $412.9 \%$ & (15) & $223.0 \%$ & (15) \\
\hline Archetype 4 & $21(2)$ & $-17.8 \%$ & (19) & $-65.2 \%$ & (18) & $-71.6 \%$ & (16) \\
\hline Archetype 5 & $10(5)$ & $20.6 \%$ & (5) & $66.2 \%$ & (5) & $52.8 \%$ & (4) \\
\hline Sample & 131 (19) & $16.1 \%$ & (112) & $152.2 \%$ & (105) & $211.3 \%$ & (92) \\
\hline
\end{tabular}

Fig. 5 Calculations of the tokens' and the archetype's mean return rates after the ICO (1 month, 6 months, and 12 months)

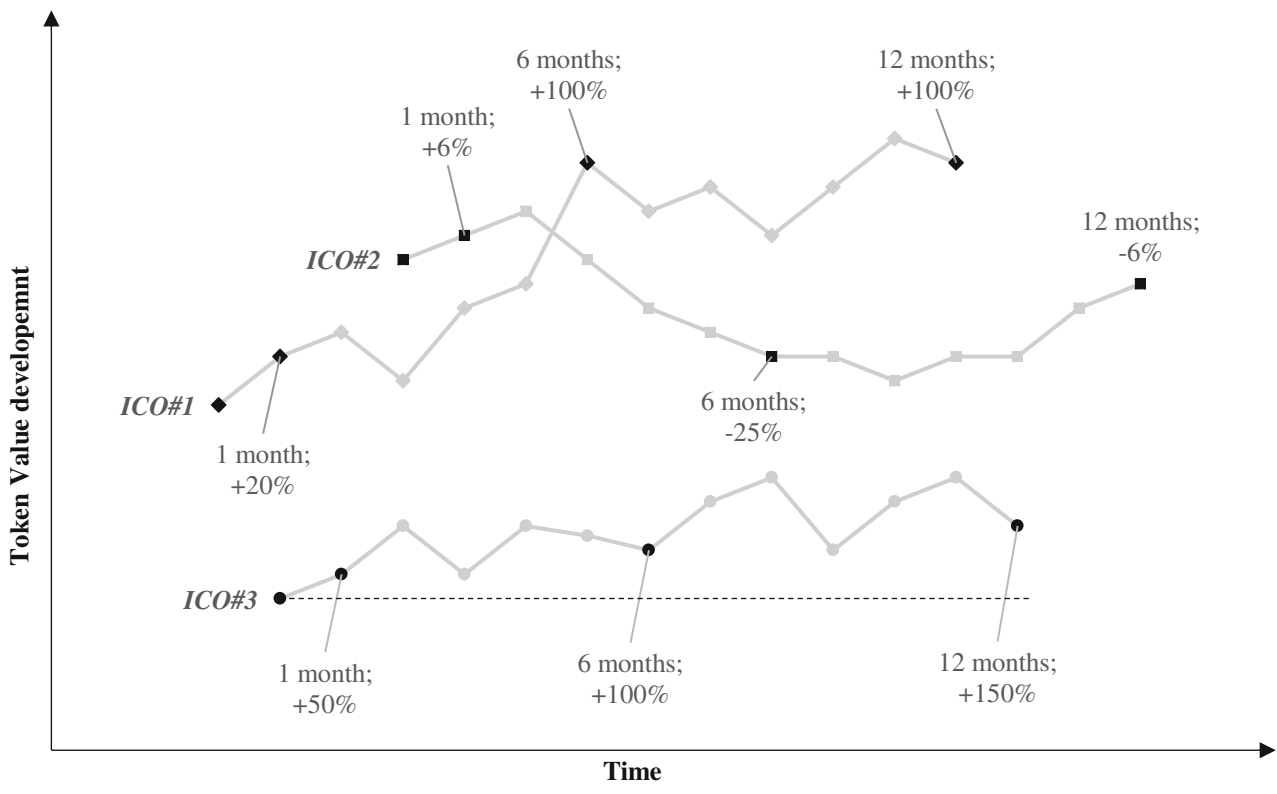


Table 6 Return rates per archetype and the corresponding return rates for bitcoin and ethereum

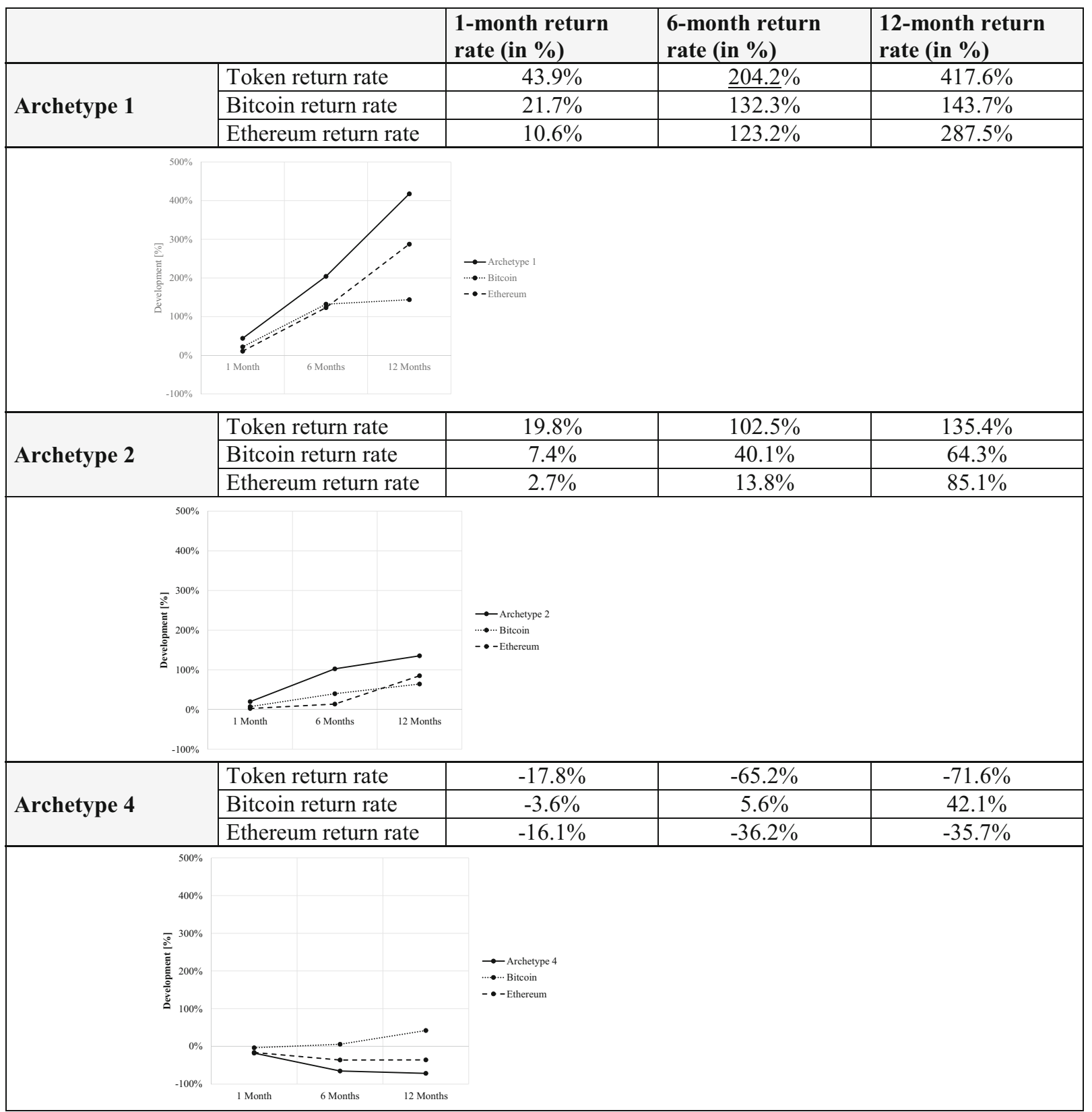

number of ICOs that indicated better development than Bitcoin or Ethereum. Further, we compared each ICO to our sample's mean return rate, and again counted the number of ICOs in each archetype that had higher or lower return rates than our sample mean. Table 7 summarizes the percentage of each archetype's ICOs that performed better than Bitcoin, Ethereum, and the entire sample.

The results revealed that between $12 \%$ and $34 \%$ of the Archetype 1 ICOs and between $25 \%$ and $39 \%$ of the Archetype 2 ICOs obtained better average return rates than
Bitcoin, Ethereum, or the entire sample, depending on the interval length, and with decreasing proportions over time. Overall, throughout all intervals and all comparison measures, Archetype 2 indicated higher proportions of overperforming ICOs than Archetype 1. Similar to archetypes 1 and 2, the proportion of overperforming ICOs of Archetype 4 decreased over time. For this archetype, all ICOs' return rates in the 6month and 12-month intervals were below the sample's average. 
Table 7 The proportion of ICOs per archetype that performed better than bitcoin, ethereum, and the overall sample

1 -month relative value (in \%) and 6-month relative value (in \%) and 12 -month relative value (in \%) the absolute number the absolute number and the absolute number

\begin{tabular}{|c|c|c|c|c|c|c|c|}
\hline \multirow[t]{3}{*}{ Archetype 1 compared to... } & Bitcoin & $30.8 \%$ & $12 / 39$ & $24.3 \%$ & $9 / 37$ & $12.1 \%$ & $4 / 33$ \\
\hline & Ethereum & $34.2 \%$ & $13 / 38$ & $22.2 \%$ & $8 / 36$ & $21.9 \%$ & $7 / 32^{\mathrm{a}}$ \\
\hline & Entire sample & $25.6 \%$ & $10 / 39$ & $18.9 \%$ & $7 / 37$ & $12.1 \%$ & $4 / 33$ \\
\hline \multirow[t]{3}{*}{ Archetype 2 compared to... } & Bitcoin & $35.3 \%$ & $12 / 34$ & $26.7 \%$ & $8 / 30$ & $20.8 \%$ & $5 / 24$ \\
\hline & Ethereum & $38.2 \%$ & $13 / 34$ & $30.0 \%$ & $9 / 30$ & $25.0 \%$ & $6 / 24$ \\
\hline & Entire sample & $35.3 \%$ & $12 / 34$ & $20.0 \%$ & $6 / 30$ & $16.7 \%$ & $4 / 24$ \\
\hline \multirow[t]{3}{*}{ Archetype 4 compared to... } & Bitcoin & $21.1 \%$ & $4 / 19$ & $11.1 \%$ & $2 / 18$ & $6.3 \%$ & $1 / 16$ \\
\hline & Ethereum & $36.8 \%$ & $7 / 19$ & $27.8 \%$ & $5 / 18$ & $18.8 \%$ & $3 / 16$ \\
\hline & Entire sample & $10.5 \%$ & $2 / 19$ & $0.0 \%$ & $0 / 18$ & $0.0 \%$ & $0 / 18$ \\
\hline
\end{tabular}

${ }^{a}$ For listings in 2013 or 2014, a comparison to the Ethereum return rate was not possible (Ethereum available starting on 30.07.2015)

\section{Discussion}

We will now first discuss the results for each archetype separately; second, we will provide anecdotal evidence to visualize each archetype; third, we will combine the insights and will discuss the implications in a broader context.

\section{Archetype 1: the visionary ICO}

Archetype 1 (see Table 8 ) is one of the two large (42 cases) clusters in the sample. It may offer multifaceted value propositions for investors who are truly interested in the issuer's business development and who are willing to engage in the initiative. The archetype's implementation level is mostly on-chain (83\%), with only few cases with native (14\%) or sidechain solutions (2\%). Two-thirds of the ICOs had proposed usage tokens (67\%), followed by staking tokens common (19\%). In nearly all cases (93\%), the development team received a minority of the tokens, and only for $76 \%$ of the cases was there a single or multiple lockup period, securing the long-term pursuit of objectives. Further, the tokens were not immediately distributed to the buyers after the ICO. Generally, distribution deferrals and lockups prevent the resale of tokens directly after the closing of the ICO, which stabilizes the post-ICO token price (Lee et al. 2018). Token supply growth was predominantly fixed $(83 \%)$ and the supply was capped $(86 \%)$. Usually, registration is needed (76\%), and the time horizon for the sale was set to a fixed date $(81 \%)$. A private or public pre-sale $(83 \%)$ allowed the issuer to raise funding prior to the regular sale. The team can then focus on developing the product early, whereas the early investors benefit from a discount. Thus, we conclude that this ICO archetype goes beyond being just a funding mechanism, but targets investors that truly believe in the business model and in its long-term success.

The overall average return rate of the visionary ICO archetype was the best of all the archetypes - it ranged from $43.8 \%$, to $204.2 \%$, to $417.6 \%$ for the 1 -month, 6-month, and 12 month periods. Further insights on the influences of characteristics regarding the ICO campaign, the venture, or technology capabilities on the raised amount of funding are discussed by Fisch (2019).

\section{Anecdotal evidence: SALT lending (SALT)}

The SALT lending platform allows users of blockchain assets to lever their holdings as collateral for cash loans. It is the first assetbacked lending platform to give blockchain asset holders access to liquidity without them having to sell their tokens. Thus, it bridges the gap between crypto-assets and conventional assets. The SALT ICO distributed a usage token, with fixed token supply growth and a capped token supply. Token holders have no voting rights. The token share for the team is minor and the team lockup period is set to a single period. There was a pre-sale with discount before the ICO.

\section{Archetype 2: the average ICO}

Archetype 2 (see Table 9) represents the other large cluster (42 cases) in our sample. Based on its characteristics, which resemble the patterns of a traditional crowdfunding campaign, we perceive this archetype as the most typical (average) one when considering ICOs as a novel funding approach. Based on an existing blockchain, the issuer raises a capped amount of funding $(98 \%)$ for mostly on-chain $(86 \%)$ usage tokens (74\%), staking tokens $(12 \%)$, or funding tokens $(7 \%)$. Tokens are immediately distributed after the ICO. Further, the announcement of a clear funding target range (83\%) conveys the message that the issuer intends to raise an amount aligned with the expected network development costs (Buterin 2017). In many cases, the whitepaper specifies that all funds are returned to investors if the ICO fails to reach the soft cap (Amsden and Schweizer 2018). This design characteristics reduces the investor's risks and indicates that the team 
Table 8 The distribution of characteristics within each dimension for Archetype $1(n=42)$

\begin{tabular}{|c|c|c|c|c|c|}
\hline Dimension & Characteristics & & & & \\
\hline Token implementation level & on-chain $(83 \%)$ & & native (14\%) & & sidechain $(2 \%)$ \\
\hline Token purpose/type & usage $(67 \%)$ & work $(7 \%)$ & funding $(7 \%)$ & staking $(19 \%)$ & non-equity $(0 \%)$ \\
\hline Token supply growth & fixed $(83 \%$ & & adaptive inflation ( & $10 \%)$ & fixed inflation (7\%) \\
\hline Token supply cap & capped $(86 \%)$ & & & uncapped (14\%) & \\
\hline Token burning & yes $(12 \%)$ & & & no $(88 \%)$ & \\
\hline Token distribution deferral & yes $(71 \%)$ & & & no $(29 \%)$ & \\
\hline Token holder voting rights & yes $(17 \%)$ & & & no $(83 \%)$ & \\
\hline Issuing legal structure & foundation $(14 \%)$ & & & limited $(86 \%)$ & \\
\hline Team company token share & minority $(93 \%)$ & & majority $(2 \%)$ & & half $(5 \%)$ \\
\hline Team lockup period & no $(24 \%)$ & & single period $(45 \%)$ & & multiple $(31 \%)$ \\
\hline Pre-sale before ICO & no $(17 \%)$ & private $(64 \%)$ & & public $(17 \%)$ & multiple $(2 \%)$ \\
\hline Pre-sale discount & yes $(76 \%)$ & & & no $(24 \%)$ & \\
\hline Planned occurrence & multiple rounds ( 17 & $7 \%)$ & single round $(83 \%)$ & & Unspecified $(0 \%)$ \\
\hline Registration needed & yes $(76 \%)$ & & & no $(24 \%)$ & \\
\hline Eligibility restrictions & none $(45 \%)$ & geographic $(36 \%)$ & & accreditation (5\%) & multiple (14\%) \\
\hline Purchase amount limit & none $(74 \%)$ & minimum $(12 \%)$ & & maximum $(5 \%)$ & both $(10 \%)$ \\
\hline Auction mechanism & yes $(0 \%)$ & & & no $(100 \%)$ & \\
\hline Sales price & fixed $(86 \%)$ & & & floating (14\%) & \\
\hline Price fixing currency & crypto $(67 \%)$ & & & fiat $(33 \%)$ & \\
\hline Funding currency & crypto $(83 \%)$ & & both $(17 \%)$ & & \\
\hline Funding cap & none $(10 \%)$ & hard cap (64\%) & & soft cap (2\%) & multiple (24\%) \\
\hline Time horizon & block time $(10 \%)$ & & fixed date $(81 \%)$ & & open end $(10 \%)$ \\
\hline Time-based discount & none $(67 \%)$ & & single rate $(14 \%)$ & & multiple rates $(19 \%)$ \\
\hline
\end{tabular}

closely links its funding to the development costs. Since this archetype does not transfer voting rights or company shares to the token holders $(74 \%)$, it tends to target investors who are interested in the de facto use case (i.e., the access to a provided service or platform) rather than for instance investment returns or decision rights.

Archetype 2's average return rate was positive for all three intervals and increased from the 1-month to the 6-month to the 12-month interval. As noted, Archetype 2 focuses on a collaborative setup and fairness (lockup period, return of funds in case of failure). Thus, for both the issuer and investor, the ICO is directed toward long-term success rather than very high short-term return rates. Especially in cases where issuers and investors want to closely work together, this archetype seems a good fit.

\section{Anecdotal evidence: BLOCKv (VEE)}

The BLOCKv platform enables the creation and emission of crypto-objects on a blockchain. The VEE token serves as a utility token to fuel any transaction on the platform. The tokens are implemented as ERC20 tokens, and the contract caps the total supply of VEE tokens. The tokens first get sold in a pre-sale, followed by a main sale, without a specific upper or lower limit of the purchase amount. Both sales are capped and have a fixed time horizon. The tokens allocated to the team are locked for multiple periods.

\section{Archetype 3: the liberal ICO}

Differences regarding the technical token terms predominantly characterize Archetype 3 (16 cases) (see Table 10), since it covers on-chain $(63 \%)$ and native $(38 \%)$ tokens. While many tokens use the ERC20 token standard from the Ethereum blockchain, native ICOs distribute tokens that are native to their own blockchain. These tokens are often referred to as protocol tokens. They can be used as simple currency and in other use cases, such as a stake to participate in a network (19\%). The developers often seek to create novel use cases based on these tokens. These innovative features appear to aim at overcoming challenges of existing blockchain solutions, such as scalability (Porru et al. 2017). The ICOs show comparably less governance from issuers regarding sales terms and issuer terms. Archetype 3 seeks to maximize the target group of prospective buyers, since it does not require prior registration $(100 \%)$, has no eligibility restrictions 


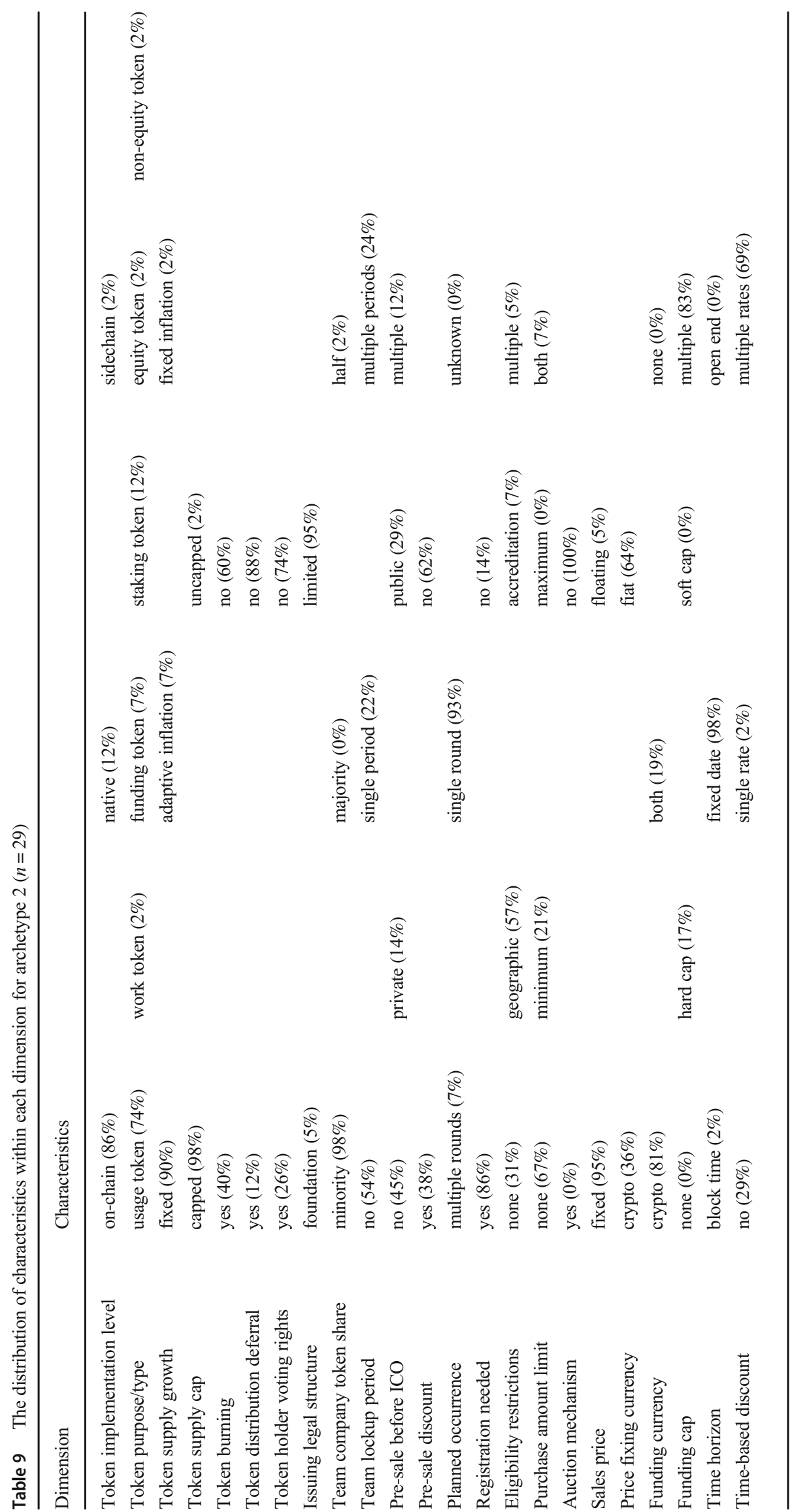


Table 10 The distribution of characteristics within each dimension for archetype $3(n=16)$

\begin{tabular}{|c|c|c|c|c|c|}
\hline Dimension & Characteristics & & & & \\
\hline $\begin{array}{l}\text { Token implementation } \\
\text { level }\end{array}$ & on-chain $(63 \%)$ & & native $(38 \%)$ & & sidechain $(0 \%)$ \\
\hline Token purpose/type & $\begin{array}{l}\text { usage token } \\
\quad(63 \%)\end{array}$ & $\begin{array}{l}\text { work token } \\
\quad(13 \%)\end{array}$ & $\begin{array}{l}\text { funding token } \\
\quad(6 \%)\end{array}$ & $\begin{array}{l}\text { staking token } \\
(19 \%)\end{array}$ & $\begin{array}{l}\text { non-equity token } \\
\quad(0 \%)\end{array}$ \\
\hline Token supply growth & fixed $(69 \%)$ & & adaptive inflation (1 & $13 \%)$ & fixed inflation (19\%) \\
\hline Token supply cap & capped $(69 \%)$ & & & uncapped $(31 \%)$ & \\
\hline Token burning & yes $(6 \%)$ & & & no $(94 \%)$ & \\
\hline Token distribution deferral & yes $(50 \%)$ & & & no $(50 \%)$ & \\
\hline Token holder voting rights & yes $(38 \%)$ & & & no $(63 \%)$ & \\
\hline Issuing legal structure & foundation $(50 \%)$ & & & limited $(50 \%)$ & \\
\hline $\begin{array}{l}\text { Team company token } \\
\text { share }\end{array}$ & minority $(88 \%)$ & & majority $(13 \%)$ & & half $(0 \%)$ \\
\hline Team lockup period & no $(81 \%)$ & & single period $(6 \%)$ & & multiple periods (13\%) \\
\hline Pre-sale before ICO & no $(88 \%)$ & private $(13 \%)$ & & public $(0 \%)$ & multiple $(0 \%)$ \\
\hline Pre-sale discount & yes $(13 \%)$ & & & no $(88 \%)$ & \\
\hline Planned occurrence & multiple rounds $\left(31^{\mathrm{C}}\right.$ & $1 \%)$ & single round $(56 \%)$ & & Unknown (13\%) \\
\hline Registration needed & yes $(0 \%)$ & & & no $(100 \%)$ & \\
\hline Eligibility restrictions & none $(100 \%)$ & geographic $(0 \%)$ & & accreditation $(0 \%)$ & multiple $(0 \%)$ \\
\hline Purchase amount limit & none $(81 \%)$ & minimum $(19 \%)$ & & maximum $(0 \%)$ & both $(0 \%)$ \\
\hline Auction mechanism & yes $(6 \%)$ & & & no $(94 \%)$ & \\
\hline Sales price & fixed $(81 \%)$ & & & floating (19\%) & \\
\hline Price fixing currency & crypto $(81 \%)$ & & & fiat $(19 \%)$ & \\
\hline Funding currency & crypto $(94 \%)$ & & both $(6 \%)$ & & none $(0 \%)$ \\
\hline Funding cap & none $(56 \%)$ & hard cap $(45 \%)$ & & soft cap $(5 \%)$ & multiple (30\%) \\
\hline Time horizon & block time $(25 \%)$ & & fixed date $(63 \%)$ & & open end $13 \%$ ) \\
\hline Time-based discount & no $(44 \%)$ & & single rate $(6 \%)$ & & multiple rates $(50 \%)$ \\
\hline
\end{tabular}

$(100 \%)$, and does not come with a purchase amount limit $(81 \%)$. This indicates that the tokens are sold liberally on a first-come, first-served basis without favoring wealthy or institutional investors. The ICO issuer grants voting rights to its investors in some cases (37\%); thus, these investors can participate and influence the ongoing development of the initiative. Another peculiarity of this archetype is the structuring of the issuing legal entity as the foundation for half the cases. Also, this archetype partially includes ICOs planning multiple funding rounds (44\%) instead of just a single round, thereby allowing for additional financing rounds when needed. This is similar to venture capital practices, where funding traditionally takes place in multiple rounds: after the seed round, the founders can prove their concept before raising additional funds for company growth (Davila et al. 2003). A consequence is that the issuing team remains incentivized to achieve ongoing success, since it directly affects subsequent funding rounds. This is why blockchain experts also believe that an iterative funding approach could be the future of ICOs (Petrovcic 2017).

Archetype 3's characteristics are very liberal, since the ICO gets along with close to no restrictions (no registration; no pre- sale; no purchase amount limit; first-come, first-served). This is particularly interesting, since our empirical analysis indicated that liberal and less regulated ICOs reveal better development on average, and thus the higher indication for success, which stands in contrast to the current public opinion that calls for stronger regulation. The results suggest that the liberal idea behind ICOs to provide open, global, and decentralized access to funding is successful. Even at this early stage, liberal ICOs are able to retain the inherent value proposition and offer an alternative to conventional funding mechanisms.

\section{Anecdotal evidence: Golem (GNT)}

Golem is a decentralized supercomputer that can be accessed by anyone. The system consists of the combined power of users' machines, from personal PCs to entire datacenters. Golem uses an Ethereum-based transaction system to clear payments. It is the first truly decentralized supercomputer and creates a market for computing power by connecting computers in a peer-to-peer network. Golem's ICO was liberal, with no token burning, no distribution deferral, no pre-sale, and no eligibility restriction. 


\section{Archetype 4: the compliant ICO}

The prevailing pattern in Archetype 4 (21 cases) (see Table 11) represents the regulatory orientation of the ICO design. By burning the unsold on-chain tokens $(100 \%)$ post-ICO $(81 \%)$, the issuer keeps the token allocation percentages between the issuer and the investors stable. Usually, the token burning benefits the token holders, since it decreases the total number of available tokens, and thus may increase the value of each individual token (Ferrara 2017). In 100\% of the ICOs, the token supply is capped. Regarding the sales terms, the issuer has more information and more control over the investors, since they need to register before they can purchase tokens (95\%). Additionally, pre-defined purchase limits restrict the token sale (minimum 67\%, minimum and maximum 19\%). Defining a minimum purchase amount can prevent a fragmentation of the token ownership, while limiting the maximum purchase amount can enhance a wider distribution of the tokens, preventing a token concentration.

The average return rate of Archetype 4 ICOs was negative for the short-term, medium-term, and long-term intervals.
Whereas in the short-term interval, more than $20 \%$ of the Archetype 4 ICOs still had higher return rates than Bitcoin or Ethereum, this proportion strongly decreased throughout the medium and long terms. Further, none of the Archetype 4 ICOs developed better than the sample's average return rate after 6 months. Compared to the Archetypes 1 and 2, that focused on a positive and specific issuer and investor collaboration, or Archetype 3, that gets along with a very liberal setup, the design of a very restrictive ICO seems to have drawbacks. Thus, we conclude that the design of ICO Archetype 3, more than others, considers the current regulatory uncertainty and seeks to comply with potential upcoming ICO regulations. This is also in line with several who warn of the downsides of the strict regulating of ICOs (Amsden and Schweizer 2018; Li and Mann 2018).

\section{Anecdotal evidence: 0xcert (ZXC)}

0xcert is a framework with a set of on-chain and off-chain rules for managing Xcerts and other standard nonfungible tokens. Xcerts represent opinionated nonfungible tokens, which also hold an imprint of an asset. With the 0xcert

Table 11 The distribution of characteristics within each dimension for archetype $4(n=21)$

\begin{tabular}{|c|c|c|c|c|c|}
\hline Dimension & Characteristics & & & & \\
\hline $\begin{array}{l}\text { Token implementation } \\
\text { level }\end{array}$ & on-chain $(90 \%)$ & & native $(0 \%)$ & & sidechain $(5 \%)$ \\
\hline Token purpose/type & $\begin{array}{l}\text { usage token } \\
\quad(76 \%)\end{array}$ & $\begin{array}{l}\text { work token } \\
\quad(14 \%)\end{array}$ & $\begin{array}{l}\text { funding token } \\
\quad(10 \%)\end{array}$ & $\begin{array}{l}\text { staking token } \\
\quad(0 \%)\end{array}$ & $\begin{array}{l}\text { non-equity token } \\
\quad(0 \%)\end{array}$ \\
\hline Token supply growth & fixed $(86 \%)$ & & adaptive inflation (10 & $0 \%)$ & fixed inflation $(5 \%)$ \\
\hline Token supply cap & capped $(100 \%)$ & & & uncapped $(0 \%)$ & \\
\hline Token burning & yes $(81 \%)$ & & & no $(19 \%)$ & \\
\hline Token distribution deferral & yes $(81 \%)$ & & & no $(19 \%)$ & \\
\hline Token holder voting rights & yes $(14 \%)$ & & & no $(86 \%)$ & \\
\hline Issuing legal structure & foundation $(24 \%)$ & & & limited $(76 \%)$ & \\
\hline $\begin{array}{l}\text { Team company token } \\
\text { share }\end{array}$ & minority $(95 \%)$ & & majority $(0 \%)$ & & half $(5 \%)$ \\
\hline Team lockup period & no $(24 \%)$ & & single period $(29 \%)$ & & multiple periods ( $48 \%$ ) \\
\hline Pre-sale before ICO & no $(10 \%)$ & private $(43 \%)$ & & public $(29 \%)$ & multiple (19\%) \\
\hline Pre-sale discount & yes $(81 \%)$ & & & no $(19 \%)$ & \\
\hline Planned occurrence & multiple rounds $(5 \%$ & & single round $(95 \%)$ & & Unknown $(0 \%)$ \\
\hline Registration needed & yes $(95 \%)$ & & & no $(5 \%)$ & \\
\hline Eligibility restrictions & none $(29 \%)$ & geographic $(67 \%)$ & & $\begin{array}{l}\text { accreditation } \\
(0 \%)\end{array}$ & multiple $(5 \%)$ \\
\hline Purchase amount limit & none $(14 \%)$ & minimum $(67 \%)$ & & maximum $(0 \%)$ & both $(19 \%)$ \\
\hline Auction mechanism & yes $(0 \%)$ & & & no $(100 \%)$ & \\
\hline Sales price & fixed $(95 \%)$ & & & floating $(5 \%)$ & \\
\hline Price fixing currency & crypto $(62 \%)$ & & & fiat $(38 \%)$ & \\
\hline Funding currency & crypto $(81 \%)$ & & both $(19 \%)$ & & none $(0 \%)$ \\
\hline Funding cap & none $(0 \%)$ & hard cap (14\%) & & soft cap $(5 \%)$ & multiple $(81 \%)$ \\
\hline Time horizon & block time $(0 \%)$ & & fixed date $(100 \%)$ & & open end $(0 \%)$ \\
\hline Time-based discount & no $(14 \%)$ & & single rate $(19 \%)$ & & multiple rates $(67 \%)$ \\
\hline
\end{tabular}


protocol, one can validate proof of existence, authenticity, and ownership of these digital assets without third-party involvement. 0xcert offers the ZXC usage token. These are fungible tokens that comply with Ethereum's ERC-20 standard. 0xcert is an open-source project that strives to be community-driven, and a decentralized governance model can also be introduced. The 0xcert ICO was restrictive in its characteristics. Token supply growth was fixed, and token supply was capped. The remaining tokens were burnt, and registration was needed prior to the sale.

\section{Archetype 5: the fundraising ICO}

The number of ICOs in Archetype 5 (see Table 12) was fairly low. Differences regarding the sales terms predominantly characterize Archetype 5 (10 cases). The issuing legal organization is limited in $90 \%$, and the team receives minority token shares $(100 \%)$. The token price is fixed in fiat currency, which can be expected, owing to less fluctuation than cryptocurrencies. While a minimum contribution was set in $60 \%$ of the ICOs, no eligibility criteria restricted the participation in $80 \%$ of the cases. At the same time, the issuer accepts both fiat currency and cryptocurrencies, whereby they may ease the participation in the ICO to crypto-novices. This may further be sponsored via a discounted public pre-sale $(70 \%)$. Further, for most Archetype 5 ICOs, the issuer offers time-based discounts (single rate $10 \%$, multiple rates $70 \%$ ). There is a hard funding cap $(70 \%)$, and the time horizon is fixed $(80 \%)$. Nonetheless, owing to our very small cluster size, we opted not to go into further performance analysis for this archetype, leaving it as an interesting subject to future research.

\section{Anecdotal evidence: Tradelize (TDZ)}

Tradelize provides an ecosystem and platform for the trading of crypto-assets. Users can spend their tokens as an internal means of payment to access the platform's services. During the ICO, the token is priced at $1 \$$, with multiple discount rates during the pre-sale as well as during the main sale. The issuers installed a hard cap and asked for a minimum contribution by investors. While the distribution of the tokens to the investors was deferred for two weeks, no lockup periods applied to the team.

Table 12 Distribution of characteristics within each dimension for archetype $5(n=10)$

\begin{tabular}{|c|c|c|c|c|c|}
\hline Dimension & Characteristics & & & & \\
\hline $\begin{array}{l}\text { Token implementation } \\
\text { level }\end{array}$ & on-chain $(80 \%)$ & & native $(20 \%)$ & & sidechain $(0 \%)$ \\
\hline Token purpose/type & $\begin{array}{l}\text { usage token } \\
\quad(80 \%)\end{array}$ & $\begin{array}{l}\text { work token } \\
\quad(0 \%)\end{array}$ & $\begin{array}{l}\text { funding token } \\
\quad(10 \%)\end{array}$ & $\begin{array}{l}\text { staking token } \\
\quad(10 \%)\end{array}$ & $\begin{array}{l}\text { non-equity token } \\
(0 \%)\end{array}$ \\
\hline Token supply growth & fixed $(80 \%)$ & & adaptive inflation (10 & $\%)$ & fixed inflation $(10 \%)$ \\
\hline Token supply cap & capped $(80 \%)$ & & & uncapped $(20 \%)$ & \\
\hline Token burning & yes $(30 \%)$ & & & no $(70 \%)$ & \\
\hline Token distribution deferral & yes $(20 \%)$ & & & no $(80 \%)$ & \\
\hline Token holder voting rights & yes $(0 \%)$ & & & no $(100 \%)$ & \\
\hline Issuing legal structure & foundation $(10 \%)$ & & & limited $(90 \%)$ & \\
\hline $\begin{array}{l}\text { Team company token } \\
\text { share }\end{array}$ & minority $(100 \%)$ & & majority $(0 \%)$ & & half $(0 \%)$ \\
\hline Team lockup period & no $(30 \%)$ & & single period $(20 \%)$ & & multiple periods $(50 \%)$ \\
\hline Pre-sale before ICO & no $(20 \%)$ & private $(0 \%)$ & & public $(70 \%)$ & multiple $(10 \%)$ \\
\hline Pre-sale discount & yes $(80 \%)$ & & & no $(20 \%)$ & \\
\hline Planned occurrence & multiple rounds $(30$ & $0 \%)$ & single round $(70 \%)$ & & Unknown $(0 \%)$ \\
\hline Registration needed & yes $(40 \%)$ & & & no $(60$ & \\
\hline Eligibility restrictions & none $(80 \%)$ & geographic (10\%) & & accreditation $(0 \%)$ & multiple $(10 \%)$ \\
\hline Purchase amount limit & none $(20 \%)$ & minimum $(60 \%)$ & & maximum $(10 \%)$ & both $(10 \%)$ \\
\hline Auction mechanism & yes $(0 \%)$ & & & no $(100 \%)$ & \\
\hline Sales price & fixed $(70 \%)$ & & & floating $(30 \%)$ & \\
\hline Price fixing currency & crypto $(20 \%)$ & & & fiat $(80 \%)$ & \\
\hline Funding currency & crypto $(70 \%)$ & & both $(30 \%)$ & & none $(0 \%)$ \\
\hline Funding cap & none $(0 \%)$ & hard cap $(70 \%)$ & & soft cap $(0 \%)$ & multiple $(30 \%)$ \\
\hline Time horizon & block time $(10 \%)$ & & fixed date $(80 \%)$ & & open end $(10 \%)$ \\
\hline Time-based discount & no $(20 \%)$ & & single rate $(10 \%)$ & & multiple rates $(70 \%)$ \\
\hline
\end{tabular}




\section{Key findings}

Our three research phases, i.e., taxonomy development, cluster analysis, and performance analysis of the ICO archetypes, allow us to derive three key findings:

\section{1) A taxonomy provides a structure for ICOs}

To answer research question 1 , we focused on the identification and evaluation of ICO design parameters. To achieve this, we followed the taxonomy development method of Nickerson et al. (2013) and proposed a taxonomy for ICOs that has 23 dimensions and 66 characteristics and therefore integrates the relevant proportion of the necessary ICO design parameters. For both primary and secondary data, no further dimensions or characteristics were necessary. The taxonomy describes ICOs in detail, and can be expanded further if necessary (e.g., owing to changes in the ICO market). Further, the taxonomy depicts an explanatory artifact that helps one to understand the details in the ICO market.

\section{2) Archetypes cluster similar kinds of ICOs}

Building on the findings of research questions 1 and 2, we applied a clustering approach to identify five ICO archetypes. The five archetypes differ concerning value propositions, target groups, and existing challenges. We clustered ICOs that follow a visionary idea, feature the average crowdfunding idea, reveal liberal characteristics, show especially compliant setups, or a fundraising enclosed scope. Further, we examined these clusters and presented a qualitative interpretation for each archetype. We were able to classify existing real-world cases to one of our five archetypes and identified withincluster similarities. For investors and founders who are interested in proposing an ICO, understanding the archetypes can be a great starting point for future endeavors.

3) Indicative performance analysis provides an outlook on ICO development

Based on our understanding of the ICO market, we combined our results from research phases 1 and 2 with secondary data and provided an outlook on ICO and archetype development. We also accounted for market specificities. Further, we differentiate between archetypes and compare the average ICO performance in the short, medium, and long terms.

Overall, our findings incorporate important aspects in the discussion about ICOs generally, their potential to become a commodity funding alternative, and the regulation of ICOs. Besides the need for regulation to protect investors, the issuer needs a certain ability and freedom to determine the conditions of an ICO. This might enable the issuer to conceptualize an ICO that fits both the issuer and the investors. Consequently, this freedom could create the opportunity to incorporate collaboration between issuer and investor that goes beyond the financial aspect and contributes to longterm success of a blockchain business model. Based on our findings, we expect that the design and the regulation of ICOs might require the breaking of new ground and may include some uncertainty. However, according to our findings, it also leads to more successful ICOs and ultimately to better funding for novel and innovative ideas, which then support the economy and society.

\section{Conclusion and outlook}

An ICO as a novel funding mechanism represents a very promising example of a blockchain use case that has recently drawn much attention in both research and practice. Although first research projects analyzed specific aspects of the emerging phenomenon, we still have a poor understanding of the implications of ICOs. In this research paper, we bridged this gap and investigated ICOs concerning their design parameters, predominant archetypes, and their short-term and longterm token value developments.

Before outlining our contributions to both research and practice, we will acknowledge limitations and will highlight promising starting points for future research. First, we limited our sampling procedure to ICOs with exhaustive data available so as to allow for comprehensive structuring according to the taxonomy's dimensions and characteristics. Owing to the high effort of the data collection, the small sample size limits the generalizability of our results. This affects the taxonomy, and, consequently, the clusters as well as the results in research phase 3. Thus, future research should focus on approaches that allow for an exhaustive inclusion of ICO cases, and should even seek to focus on subsets where all cases are included in the analysis. A valid approach could also be to condense the research question, so that more ICOs can be included. Note, however, that although we excluded some ICOs, our definition of success and the study perspective were dedicated to including all the available data. Nonetheless, our manual data gathering approach enabled this research to draw first conclusions form real-world data. Second, we only addressed ICO design parameters, rather than other ICO aspects which have been examined in previous crowdfunding literature, such as the business model, industry, or the quality of marketing. However, since our focus was on deriving archetypes, our results constitute a valid and enlightening first approach toward the goal of understanding ICO patterns. Nonetheless, these aspects could be subject to further research that may help us to better understand the ICO phenomenon. Third, the ICO market is very dynamic and most ICO issuers are startups. Token sale models are constantly evolving, 
leading to dynamic emergences of novel ICO design patterns. In this context, we further acknowledge that the identification of the five archetypes was limited by the selected sample, and the addition of new ICOs to the sample and to the clustering could result in slightly modified archetypes. However, since the ICO market is constantly changing, our research reflects current developments in the ICO market. Fourth, clustering methodology has certain natural limitations (Hair et al. 2013) since it produces a non-inferential solution which heavily depends on the selection of the clustering variables, similarity measures and algorithms (Balijepally et al. 2011). However, we are confident that our chosen methodology reduces the danger of producing unstable results, since we apply the recommended two-stage clustering (e.g., Balijepally et al. 2011), and since our clustering variables "emanate from past research [... and are] consistent with the objectives of the study" (Balijepally et al. 2011, p. 377).

Our theoretical contributions addressed the research gap in four ways: First, we have provided a systematic overview over predominant ICO designs. Thus, we suggested five ICO archetypes with different value propositions, target groups, and challenges. These archetypes abstract from single peculiarities of specific ICOs, enabling generalizable propositions. Second, the archetypes extend existing classifications of ICOs by various aspects and allow for generalizable findings, instead of considering single characteristics. Third, we have laid the foundation for further research in the area of ICOs. Since the archetypes were theoretically grounded on an existing taxonomy and were empirically verified, they provide a more systematic and in-depth perspective on the phenomenon. This will help to synthesize research into ICOs and opens future promising research avenues. Further, we have built on the existing knowledge and have combined different approaches in order to provide an outlook on the ICO and archetype development on the secondary market. Future research can build on these insights and can propose additional research projects. Fourth, our findings of ICO archetypes are crucial for the research into ICO and blockchain governance issues, since they allow one to derive the impacts of different governance configurations.

Moreover, our research provides practitioners with various backgrounds and perspectives on the ICO phenomenon. First, with our proposed taxonomy, we provide a classification scheme that allows one to comprehensibly structure this complex domain. Second, the classification into predominant archetypes may provide structured guidance for ventures that plan to conduct an ICO. Thus, our taxonomy and archetypes allow one to reduce complexity in the heterogeneous ICO market. Third, from an investor perspective, the archetypes can lead to more informed and grounded investment decisions. Additionally, for traditional financial intermediaries, including early-stage venture capitalists or crowdfunding platforms, the taxonomy and archetypes may help to characterize potential competitors. Our analysis of the ICO archetypes may help regulators and government institutions to perform regulatory tasks more effectively.

Funding Open Access funding enabled and organized by Projekt DEAL. Supported by PayPal and the Luxembourg National Research Fund FNR, Luxembourg (P17/IS/13342933/PayPal-FNR/Chair in DFS/ Gilbert Fridgen).

\section{Appendix}

\begin{tabular}{|c|c|c|}
\hline Ticker & Cluster & Issue date \\
\hline $1 \mathrm{ST}$ & 1 & 28-Sep-16 \\
\hline $3 \mathrm{DC}$ & 2 & 24-Sep-19 \\
\hline $\mathrm{ABL}$ & 2 & 14-Aug-18 \\
\hline ABYSS & 2 & 08-Jun-18 \\
\hline $\mathrm{ADB}$ & 2 & 04-Feb-18 \\
\hline $\mathrm{ADM}$ & 2 & 18-Jan-19 \\
\hline AEN & 4 & 06-Apr-19 \\
\hline AISI & 5 & no trading \\
\hline ANS & 1 & 09-Sep-16 \\
\hline ANT & 2 & 18-May-17 \\
\hline ARR & 2 & 18-Jun-19 \\
\hline AST & 1 & 17-Oct-17 \\
\hline ASTRO & 2 & 17-Nov-17 \\
\hline BAT & 3 & 01-Jun-17 \\
\hline BCK & 1 & no trading \\
\hline BITX & 4 & 01-Aug-18 \\
\hline BNT & 4 & 22-Jun-17 \\
\hline BPL & 1 & 10-Nov-17 \\
\hline BST & 2 & 05-Jun-19 \\
\hline CAN & 2 & 08-Jan-18 \\
\hline CFI & 1 & 19-Jun-17 \\
\hline $\mathrm{CHI}$ & 4 & 08-Nov-18 \\
\hline CRBT & 4 & 17-Sep-18 \\
\hline CRON & 2 & 02-Sep-19 \\
\hline CRV8 & 2 & no trading \\
\hline CSM & 1 & 27-Jul-18 \\
\hline $\mathrm{CVC}$ & 1 & 17-Jul-17 \\
\hline DACC & 2 & 27-Jul-18 \\
\hline DANK & 5 & no trading \\
\hline DATA & 1 & 03-Nov-17 \\
\hline DCT & 3 & 02-Jul-17 \\
\hline DENT & 3 & 13-Aug-17 \\
\hline DFN & 3 & no trading \\
\hline DGCT & 2 & no trading \\
\hline DGD & 3 & 18-Apr-16 \\
\hline DOOH & 2 & no trading \\
\hline
\end{tabular}


DOT

DREAM

DTX

DTx2

ELY

eMTV

EQUI

ESS

ETH

ETKN

FIL

FTM

FXP

GBT

GNO

GNT

GoC (former ELI)

GOT

GRFT

GXC

HGT

ICN

IMT

INCX

IOTA

KNC

Komodo KMD

LCS

LDX (LeadRex)

LENDO

LKK

LSK

Lunes

MAID

MANA

MAS

MASP

MCO

MET

MGX

Minter

MKR

MLN

MOD

NEU

OLT

OMG

OMNI

\section{5-Feb-15}

24-May-19

11-Jul-18

11-Jul-18

19-Jul-18

no trading

24-May-17

07-Jul-18

07-Aug-15

27-Jun-18

13-Dec-17

30-Oct-18

28-Dec-18

no trading

01-May-17

18-Nov-16

04-Aug-18

05-Jul-18

09-Mar-18

25-Jun-17

12-Oct-17

30-Sep-16

31-Aug-18

02-Aug-18

13-Jun-17

24-Sep-17

06-Feb-17

25-Jul-18

13-Mar-18

no trading

14-Nov-16

06-Apr-16

15-Mar-19

28-Apr-14

17-Sep-17

27-Sep-18

27-Sep-18

03-Jul-17

26-Jun-18

09-Nov-19

no trading

20-Dec-17

22-Feb-17

23-Oct-17

29-Dec-17

12-Jul-18

14-Jul-17

25-Dec-13
OOT

ORBS

PAY

PIX

PXLT

QBX

QNT

RDN

REP

RSK

S

SALT

SAN

Scorum

SHA

SHR

SILK

SNT

SQR

STM

STORJ

STORM

T2T

TDZ

TERN

TERN2

TEZ

TGAME

TKLN

TNG

TNT

UBT

UBX

UMT

UP

VEE

VID

VIDT

VIN

VIRT

VITO

VLR

WGP

WTL

XBASE

ZRX

ZXC
12-May-18

03-Apr-19

08-Jul-17

25-Sep-17

11-Jul-19

19-Jul-19

11-Aug-18

08-Nov-17

27-Oct-15

05-Dec-18

11-Oct-18

29-Sep-17

12-Jul-17

02-Sep-19

03-Apr-19

29-Nov-19

16-Oct-18

28-Jun-17

10-Jun-19

05-Oct-18

02-Jul-17

20-Dec-17

no trading

no trading

27-Jul-18

no trading

02-Oct-17

18-Jul-18

04-Sep-19

no trading

27-Aug-17

21-May-18

14-Aug-18

no trading

21-Mar-18

28-Nov-17

28-Aug-19

06-Apr-19

07-Aug-18

no trading

no trading

no trading

09-May-19

19-Oct-18

26-Mar-19

16-Aug-17

12-Jul-18 
Open Access This article is licensed under a Creative Commons Attribution 4.0 International License, which permits use, sharing, adaptation, distribution and reproduction in any medium or format, as long as you give appropriate credit to the original author(s) and the source, provide a link to the Creative Commons licence, and indicate if changes were made. The images or other third party material in this article are included in the article's Creative Commons licence, unless indicated otherwise in a credit line to the material. If material is not included in the article's Creative Commons licence and your intended use is not permitted by statutory regulation or exceeds the permitted use, you will need to obtain permission directly from the copyright holder. To view a copy of this licence, visit http://creativecommons.org/licenses/by/4.0/.

\section{References}

Adhami, S., Giudici, G., \& Martinazzi, S. (2018). Why do businesses go crypto? An empirical analysis of initial coin offerings. Journal of Economics and Business, 100, 64-75. https://doi.org/10.1016/j. jeconbus.2018.04.001.

Ahlers, G. K. C., Cumming, D., Günther, C., \& Schweizer, D. (2015). Signaling in equity Crowdfunding. Entrepreneurship Theory and Practice, 39(4), 955-980. https://doi.org/10.1111/etap.12157.

Aldenderfer, M. S., \& Blashfield, R. K. (1984). Cluster analysis. Quantitative applications in the social sciences. Beverly Hills: Sage Publication. https://doi.org/10.4135/9781412983648.

Amsden, R., \& Schweizer, D. (2018). Are Blockchain Crowdsales the new 'Gold Rush'? Success Determinants of Initial Coin Offerings. SSRN Electronic Journal. https://doi.org/10.2139/ssrn.3163849.

Arnold, L., Brennecke, M., Camus, P., Fridgen, G., Guggenberger, T., Radszuwill, S., Rieger, A., Schweizer, A., \& Urbach, N. (2019). Blockchain and initial coin offerings: Blockchain's implications for Crowdfunding. In H. Treiblmaier \& R. Beck (Eds.), Business transformation through Blockchain (pp. 233-272). Cham: Springer International Publishing.

Bachmann, N., Drasch, B., Miksch, M., \& Schweizer, A. (2019). Dividing the ICO Jungle: Extracting and Evaluating Design Archetypes. Proceedings of the 14th International Conference on Wirtschaftsinformatik. Siegen: Germany.

Bailey, K. D. (1984). A three-level measurement model. Quality \& Quantity, 18(3), 225-245. https://doi.org/10.1007/BF00156457.

Balijepally, V., Mangalaraj, G., \& Iyengar, K. (2011). Are we wielding this hammer correctly? A reflective review of the application of cluster analysis in information systems research. Journal of the Association for Information Systems, 12(5), 375-413. https://doi. org/10.17705/1jais.00266.

Beck, R., Stenum Czepluch, J., Lollike, N., \& Malone, S. (2016). Blockchain - the gateway to trust - free cryptographic transactions. In I. Benbasat, N. Bjørn-Andersen, \& A. Sencer (Eds.), Proceedings of the 24th European Conference on Information Systems (ECIS), 1-14.

Beck, R., Avital, M., Rossi, M., \& Thatcher, J. B. (2017). Blockchain technology in business and information systems research. Business \& Information Systems Engineering, 59(6), 381-384. https://doi. org/10.1007/s12599-017-0505-1.

Benedetti, H. E., \& Kostovetsky, L. (2018). Digital Tulips? Returns to Investors in Initial Coin Offerings. SSRN Electronic Journal. https:// doi.org/10.2139/ssrn.3182169.

Berkhin, P. (2006). A survey of clustering data mining techniques. In J. Kogan, C. Nicholas, \& M. Teboulle (Eds.), Grouping multidimensional data (pp. 25-71). Berlin, Heidelberg: Springer. https://doi. org/10.1007/3-540-28349-8_ 2.

Boreiko, D., \& Sahdev, N. K. (2018). To ICO or not to ICO - Empirical analysis of initial coin offerings and token sales. SSRN Electronic Journal. https://doi.org/10.2139/ssm.3209180.
Buterin, V. (2014). A next-generation smart contract and decentralized application platform. [White paper]. Ethereum. https://cryptorating. eu/whitepapers/Ethereum/Ethereum_white_paper.pdf

Buterin, V. (2017). Analyzing token sale models, 2018 (No. 07.07.2018): Buterin, Vitalik. Retrieved from https://vitalik.ca/general/2017/06/ 09/sales.html

Catalini, C., \& Gans, J. S. (2018). Initial coin offerings and the value of crypto tokens, [Working paper 24418]. NBER. https://doi.org/10. 3386/w24418.

Chanson, M., Risius, M., \& Wortmann, F. (2018). Initial coin offerings (ICOs): An introduction to the novel funding mechanism based on Blockchain technology. Proceedings of the 24th Americas Conference on Information Systems (AMCIS). New Orleans.

Clercq, D. d., \& Dimov, D. (2008). Internal knowledge development and external knowledge access in venture capital investment performance. Journal of Management Studies, 45(3), 585-612. https:// doi.org/10.1111/j.1467-6486.2007.00747.x.

Conley, J. P. (2017). Blockchain and the economics of crypto-tokens and initial coin offerings. Vanderbilt University Department of Economics Working Papers, [VUECON-17-00008]. http://www. accessecon.com/Pubs/VUECON/VUECON-17-00008.pdf

Cumming, D., Fleming, G., \& Schwienbacher, A. (2005). Liquidity risk and venture capital finance. Financial Management, 34(4), 77105. https://doi.org/10.1111/j.1755-053X.2005.tb00119.x.

Danmayr, F. (2014). Archetypes of crowdfunding platforms: Gabler Verlag. https://doi.org/10.1007/978-3-658-04559-3.

Davila, A., Foster, G., \& Gupta, M. (2003). Venture capital financing and the growth of startup firms. Journal of Business Venturing, 18(6), 689-708. https://doi.org/10.1016/S0883-9026(02)00127-1.

Dexter, L. A. (2006). Elite and specialized interviewing. ECPR classics. Colchester: ECPR Press.

Drasch, B. J., Schweizer, A., \& Urbach, N. (2018). Integrating the 'troublemakers': A taxonomy for cooperation between banks and fintechs. Journal of Economics and Business, 100, 26-42. https:// doi.org/10.1016/j.jeconbus.2018.04.002.

Drasch, B. J., Fridgen, G., Manner-Romberg, T., Nolting, F. M., \& Radszuwill, S. (2020). The token's secret: The two-faced financial incentive of the token economy. Electronic Markets, 37(3), 668567. https://doi.org/10.1007/s12525-020-00412-9.

EFSA. (2017). The legal framework of initial coin offering in Estonia. Estonian financial supervision authority. https://www.fi. ee/index.php?id=21662

Ehrsam, F. (2016). Blockchain Tokens and the dawn of the Decentralized Business Model. The Coinbase blog. https://blog.coinbase.com/appcoins-and-the-dawn-of-the-decentralized-business-model$8 \mathrm{~b} 8 \mathrm{c} 951 \mathrm{e} 734 \mathrm{f}$

Everitt, B. S., Landau, S., Leese, M., \& Stahl, D. (2011). Cluster analysis. Chichester: Wiley.

Fanning, K., \& Centers, D. P. (2016). Blockchain and its coming impact on financial services. Journal of Corporate Accounting \& Finance, 27(5), 53-57. https://doi.org/10.1002/jcaf.22179.

Ferrara, N. (2017). 'Token burning' and other crypto jargon simplified. Forbes. https://www.forbes.com/sites/eidoo/2017/11/29/tokenburning-and-other-crypto-jargon-simplified/\#2c06d5f954c4.

Finch, H. (2005). Comparison of distance measures in cluster analysis with dichotomous data. Journal of Data Science, 3, 85-100.

Fisch, C. (2019). Initial coin offerings (ICOs) to finance new ventures: An exploratory study. Journal of Business Venturing, 34(1), 1-22. https://doi.org/10.2139/ssrn.3147521.

Fridgen, G., Regner, F., Schweizer, A. \& Nils, U. (2018). Don’t slip on the ICO-A taxonomy for a blockchain-enabled form of crowdfunding. Proceedings of the 26th European Conference on Information Systems. Portsmouth, pp. 1-17.

Glaser, F. (2017). Pervasive decentralisation of digital infrastructures: A framework for Blockchain enabled system and use case analysis. Proceedings of the 50th Hawaii International Conference on 
System Sciences (HICSS). Waikoloa Village, Hawaii. https://doi. org/10.24251/HICSS.2017.186.

Glaser, F., \& Bezzenberger, L. (2015). Beyond cryptocurrencies-a taxonomy of decentralized consensus systems. Proceedings of the 23rd European Conference on Information Systems (ECIS). Germany: Münster.

Glass, R. L., \& Vessey, I. (1995). Contemporary application-domain taxonomies. IEEE Software(4), 63-76. https://doi.org/10.1109/52. 391837.

Gregor, S. (2006). The nature of theory in information systems. MIS Quarterly, 30(3), 611-642. https://doi.org/10.2307/25148742.

Haas, P., Blohm, I., \& Leimeister, J. M. (2014). An empirical taxonomy of Crowdfunding intermediaries. Proceedings of the 35th International Conference on Information Systems (ICIS). Auckland: New Zealand.

Haas, P., Blohm, I., Peters, C., \& Leimeister, J. M. (2015). Modularization of Crowdfunding services-designing disruptive innovations in the banking industry. Proceedings of the 36th International Conference on Information Systems (ICIS). Fort Worth, Texas: USA.

Hair, J. F., Black, W. C., Babin, B. J., \& Anderson, R. E. (2013). Multivariate data analysis (7th ed.). Upper Saddle River (NJ): Pearson Education.

Howell, S., Niessner, M., \& Yermack, D. (2018). Initial coin offerings: Financing growth with cryptocurrency token sales. The Review of Financial Studies, 33(9), 3925-3974. https://doi.org/10.1093/rfs/ hhz131.

Hu, A., Parlour, C. A., \& Rajan, U. (2018). Cryptocurrencies: Stylized facts on a new investible instrument. SSRN Electronic Journal. https://doi.org/10.2139/ssrn.3182113.

ICObench. (2018). https://icobench.com/

ICODATA.IO. (2019). ICO STATUS. https://www.icodata.io/stats/2018

Jöhnk, J., Röglinger, M., Thimmel, M., \& Urbach, N. (2017). How to implement agile IT setups: A taxonomy of design options. 25th European Conference on Information Systems (ECIS). Guimarães: Portugal.

Keller, R., \& König, C. (2014). A reference model to support risk identification in cloud networks. Proceedings of the 35th International Conference on Information Systems (ICIS). Auckland: New Zealand.

Ketchen, D. J., \& Shook, C. L. (1996). The application of cluster analysis in strategic management research: An analysis and critique. Strategic Management Journal, 17(6), 441-458. https://doi.org/10. 1002/(SICI)1097-0266(199606)17:6<441::AID-SMJ819>3.0.CO; $2-\mathrm{G}$.

Ketchen, D. J., Thomas, J. B., \& Snow, C. C. (1993). Organizational configurations and performance: A comparison of theoretical approaches. Academy of Management Journal, 36(6), 12781313. https://doi.org/10.5465/256812.

Kuo Chuen, D. L. (2017). Fintech tsunami: Blockchain as the driver of the fourth industrial revolution. SSRN Electronic Journal, 1-11. https://doi.org/10.2139/ssrn.2998093.

Lee, J., Li, T., \& Shin, D. (2018). The wisdom of crowds and information cascades in fintech: Evidence from initial coin offerings. SSRN Electronic Journal. https://doi.org/10.2139/ssrn.3195877.

Li, J., \& Mann, W. (2018). Initial coin offering and platform building. SSRN Electronic Journal. https://doi.org/10.2139/ssrn.3088726.

Malhotra, A., Gosain, S., \& El Sawy, O. A. (2005). Absorptive capacity configurations in supply chains: Gearing for partner-enabled market knowledge creation. MIS Quarterly, 29(1), 145-187. https://doi.org/ $10.2307 / 25148671$

Milligan, G. W., \& Sokol, L. M. (1980). A two-stage clustering algorithm with robust recovery characteristics. Educational and Psychological Measurement, 40(3), 755-759. https://doi.org/10.1177/ 001316448004000320
Mollick, E. (2014). The dynamics of crowdfunding: An exploratory study. Journal of Business Venturing, 29(1), 1-16. https://doi.org/ 10.1016/j.jbusvent.2013.06.005.

Momtaz, P. P. (2018). Initial coin offerings. PLOS ONE. https://doi.org/ 10.1371/journal.pone.0233018.

Morse, J. M. (1995). The significance of saturation. Qualitative Health Research, 5, 147-149.

Nærland, K., Müller-Bloch, C., Beck, R., \& Palmund, S. (2017). Blockchain to rule the waves - nascent design principles for reducing risk and uncertainty in decentralized environments. Proceedings of the 38th International Conference on Information Systems (ICIS). Seoul: South Korea.

Nakamoto, S. (2008). Bitcoin: A peer-to-peer electronic cash system. Bitcoin. hhttps://www.bitcoin.org/bitcoin.pdf

Nickerson, R. C., Muntermann, J., \& Varshney, U. (2010). Taxonomy development in information systems: A literature survey and problem statement. Proceedings of the the 16th American Conference on Information Systems (AMCIS). Lima: Peru.

Nickerson, R. C., Varshney, U., \& Muntermann, J. (2013). A method for taxonomy development and its application in information systems. European Journal of Information Systems, 22(3), 336-359. https:// doi.org/10.1057/ejis.2012.26.

Perks, H., Cooper, R., \& Jones, C. (2005). Characterizing the role of design in new product development: An empirically derived taxonomy. Journal of Product Innovation Management, 22(2), 111-127. https://doi.org/10.1111/j.0737-6782.2005.00109.x.

Petrovcic, D. (2017). ICOs, don't bite off more than you can chew. Hackernoon. https://hackernoon.com/icos-dont-bite-offmore-than-you-can-chew-d658aae9579e

Porru, S., Pinna, A., Marchesi, M., \& Tonelli, R. (2017). Blockchainoriented software engineering: Challenges and new directions. Proceedings of the 39th International Conference on Software Engineering Companion. Buenos Aires: IEEE Press. https://doi. org/10.1109/ICSE-C.2017.142.

Punj, G., \& Stewart, D. W. (1983). Cluster analysis in marketing research: Review and suggestions for application. Journal of Marketing Research, 20(2), 134-148. https://doi.org/10.1177/ 002224378302000204.

Püschel, L., Schlott, H., \& Röglinger, M. (2016). What's in a smart thing? Development of a multi-layer taxonomy. Proceedings of the 37th International Conference on Information Systems (ICIS). Dublin: Ireland.

Ravikant, N. (2014). The bitcoin model for crowdfunding. https:// startupboy.com/2014/03/09/the-bitcoin-model-for-crowdfunding/

Schultze, U., \& Avital, M. (2011). Designing interviews to generate rich data for information systems research. Information and Organization, 21(1), 1-16. https://doi.org/10.1016/j.infoandorg. 2010.11.001.

Schweizer, A., Schlatt, V., Urbach, N., \& Fridgen, G. (2017). Unchaining social businesses: Blockchain as the basic Technology of a Crowdlending Platform. Proceedings of the 38th International Conference on Information Systems (ICIS). Seoul: South Korea.

Sillaber, C., \& Waltl, B. (2017). Life cycle of smart contracts in Blockchain ecosystems. Datenschutz und Datensicherheit - DuD, 41(8), 497-500. https://doi.org/10.1007/s11623-017-0819-7.

Smith + Crown. (2017). Token sale market performance. https://www. smithandcrown.com/token-sale-market-performance/\#token-salemarket-performance

Soh, C., \& Markus, M. L. (2002). Business-to-business E-marketplaces: A strategic archetypes approach. Proceedings of the $23 \mathrm{rd}$ International Conference on Information Systems (ICIS). Barcelona: Spain.

Szabo, N. (1997). Smart contracts: Formalizing and securing relationships on public networks. First Monday, 2(9). https://doi.org/10. 5210/fm.v2i9.548. 
van de Vrande, V., de Jong, J. P. J., Vanhaverbeke, W., \& de Rochemont, M. (2009). Open innovation in SMEs: Trends, motives and management challenges. Technovation, 29(6-7), 423-437. https://doi.org/ 10.1016/j.technovation.2008.10.001.

Vigna, P., Shifflett, S., \& Ostroff, C. (2018). What crypto downturn? ICO fundraising surges in 2018. Wall Street Journal. https://www.wsj. com/articles/what-crypto-downturn-ico-fundraising-surges-in2018-1530466008

Williams, K., Chatterjee, S., \& Rossi, M. (2008). Design of emerging digital services: A taxonomy. European Journal of Information Systems, 17(5), 505-517. https://doi.org/10.1057/ejis.2008.38.

Xu, X., Weber, I., Staples, M., Zhu, L., Bosch, J., Bass, L., Pautasso, C., \& Rimba, P. (2017). A taxonomy of Blockchain-based systems for architecture design. International Conference on Software Architecture (IEEE) Gothenburg: IEEE. https://doi.org/10.1109/ ICSA.2017.33.
Yin, R. K. (2017). Case study research and applications: Design and methods. Thousand Oaks, CA: Sage Publications.

Zetzsche, D. A., Buckley, R. P., Arner, D. W., \& Föhr, L. (2017). The ICO gold rush: It's a scam, It's a bubble, It's a super challenge for regulators. SSRN Electronic Journal. https://doi.org/10.2139/ssrn. 3072298 .

Zohar, A. (2015). Bitcoin: Under the Hood. Communications of the ACM, 58(9), 104-113. https://doi.org/10.1145/2701411.

Publisher's note Springer Nature remains neutral with regard to jurisdictional claims in published maps and institutional affiliations. 